ESAIM: COCV 27 (2021) 100

https://doi.org/10.1051/cocv/2021088
ESAIM: Control, Optimisation and Calculus of Variations

www.esaim-cocv.org

\title{
HIGH-ORDER HOMOGENIZATION IN OPTIMAL CONTROL BY THE BLOCH WAVE METHOD*
}

\author{
Agnes Lamacz-Keymling and Irwin Yousept**๑ఠ
}

\begin{abstract}
This article examines a linear-quadratic elliptic optimal control problem in which the cost functional and the state equation involve a highly oscillatory periodic coefficient $A^{\varepsilon}$. The small parameter $\varepsilon>0$ denotes the periodicity length. We propose a high-order effective control problem with constant coefficients that provides an approximation of the original one with error $O\left(\varepsilon^{M}\right)$, where $M \in \mathbb{N}$ is as large as one likes. Our analysis relies on a Bloch wave expansion of the optimal solution and is performed in two steps. In the first step, we expand the lowest Bloch eigenvalue in a Taylor series to obtain a high-order effective optimal control problem. In the second step, the original and the effective problem are rewritten in terms of the Bloch and the Fourier transform, respectively. This allows for a direct comparison of the optimal control problems via the corresponding variational inequalities, leading to our main theoretical result on the high-oder approximation.
\end{abstract}

Mathematics Subject Classification. 35B27, 35P05, 49J20.

Received November 9, 2020. Accepted September 2, 2021.

\section{INTRODUCTION}

Many modern key technologies call for mathematical modeling through partial differential equations (PDEs) with macro- and microstructures. The simplest way to decode a microstructure is to consider periodic coefficients with a small periodicity length $\varepsilon>0$. In such a situation, the central question concerns the effective or asymptotic behavior of the problem in the homogenization limit $\varepsilon \rightarrow 0$. The periodic homogenization theory was mainly developed in the late '70s focusing on the effective behavior of elliptic PDEs (see [6, 8, 31]). Since then, the theory has been generalized to various types of equations and more complex models, including perforated domains [12], high contrast media, and singular geometries [9, 29], just to mention a few.

The homogenization theory plays as well a profound role in the optimal control of PDEs with highly oscillatory coefficients. Such problems are particularly encountered in electrical and electronic engineering applications $[34,35]$, which require a substantial extension of the developed techniques by the homogenization theory. We refer to $[11,16,19-21,23-27]$ for the interplay of homogenization and optimal control based on weak convergence methods. In Kogut and Leugering [24], the concept of variational S-homogenization was proposed and analyzed for the limiting behavior of an optimal control problem governed by a linear elliptic equation with control and state constraints. Also, they developed the principle of fictitious homogenization [23] for optimal control

${ }^{*}$ The work of the second author was funded by German Research Foundation (DFG grants YO159/2-2 and YO159/4-1).

Keywords and phrases: Optimal control, periodic homogenization, Bloch analysis.

Universität Duisburg-Essen, Fakultät für Mathematik, Thea-Leymann-Straße 9, 45127 Essen, Germany.

** Corresponding author: irwin.yousept@uni-due.de 
problems in variable domains within the framework of variational S-convergence. In [25], the homogenization of state-constrained semilinear optimal control problems was discussed and explored. From among many other contributions, we refer to the monograph by Kogut and Leugering [27] for more insights into the topic.

The previously mentioned works mainly focus on the plain convergence behavior in the limit $\varepsilon \rightarrow 0$. To the best of the authors' knowledge, only in [22] convergence rates and higher-order approximations for rapidly oscillatory optimal control problems are provided. This contribution derives and justifies the high-order asymptotics for parabolic optimal control problems with periodic coefficients. The author uses a classical two-scale approach by expanding the optimal control as well as the corresponding optimal state in a two-scale power series. This is possible, since [22] does not include additional control or state constraints, i.e., the admissible control set comprises a linear space of a certain regularity. Indeed, in the classical two-scale approach, higher-order approximations are achieved by adding highly oscillatory terms to the effective solution, the so-called correctors, which solve microscopic cell problems and characterize the oscillatory behavior of the original solution. While in the setting of [22] this strategy is particularly suitable, it seems difficult to apply the classical two-scale approach to optimal control problems with control or state constraints since adding correctors to the effective control function in general will destroy its admissibility. To circumvent this difficulty, we propose the use of a spectral method involving the so-called Bloch waves, which allows for a variety of $L^{2}$-conditions in the admissible set. Examples are provided in Section 1.2.

This paper is aimed at the high-order asymptotic behavior of a linear-quadratic elliptic optimal control problem with $\varepsilon$-periodic coefficients and $\varepsilon$-dependent admissible sets. More precisely, we consider a symmetric and uniformly elliptic coefficient matrix $A \in L^{\infty}\left(\mathbb{R}^{n} ; \mathbb{R}^{n \times n}\right)$ with $n \in \mathbb{N}$ that is periodic with respect to the unit cube $Y:=[0,1]^{n}$. Setting $A^{\varepsilon}(x):=A\left(\frac{x}{\varepsilon}\right)$, we introduce an energy functional $E^{\varepsilon}: H^{1}\left(\mathbb{R}^{n}\right) \rightarrow \mathbb{R}$ and a cost functional $J^{\varepsilon}: L^{2}\left(\mathbb{R}^{n}\right) \times H^{1}\left(\mathbb{R}^{n}\right) \rightarrow \mathbb{R}$ as follows:

$$
E^{\varepsilon}(y):=\int_{\mathbb{R}^{n}} \nabla y(x) \cdot A^{\varepsilon}(x) \nabla y(x)+|y(x)|^{2} \mathrm{~d} x
$$

and

$$
J^{\varepsilon}(u, y):=\frac{\mu_{1}}{2} E^{\varepsilon}\left(y-y_{d, 1}^{\varepsilon}\right)+\frac{\mu_{2}}{2} \int_{\mathbb{R}^{n}}\left|y(x)-y_{d, 2}^{\varepsilon}(x)\right|^{2} \mathrm{~d} x+\frac{\kappa}{2} \int_{\mathbb{R}^{n}}|u(x)|^{2} \mathrm{~d} x
$$

with fixed constants $\mu_{1}, \mu_{2} \geq 0$ and $\kappa>0$. Furthermore, $y_{d, 1}^{\varepsilon} \in H^{1}\left(\mathbb{R}^{n}\right)$ and $y_{d, 2}^{\varepsilon} \in L^{2}\left(\mathbb{R}^{n}\right)$ are given functions denoting, respectively, the desired states with respect to the energy norm and the $L^{2}\left(\mathbb{R}^{n}\right)$-norm. In view of (1.2), our focus is set on the following linear-quadratic elliptic optimal control problem:

$$
\left\{\begin{array}{l}
\min J^{\varepsilon}\left(u_{\varepsilon}, y_{\varepsilon}\right) \\
\text { over }\left(u_{\varepsilon}, y_{\varepsilon}\right) \in U_{a d}^{\varepsilon} \times H^{1}\left(\mathbb{R}^{n}\right) \\
\text { s.t. }-\nabla \cdot\left(A^{\varepsilon}(x) \nabla y_{\varepsilon}(x)\right)+y_{\varepsilon}(x)=f^{\varepsilon}(x)+u_{\varepsilon}(x) \text { in weak sense on } \mathbb{R}^{n}
\end{array}\right.
$$

In the setting of $\left(\mathrm{P}_{\varepsilon}\right), f^{\varepsilon} \in L^{2}\left(\mathbb{R}^{n}\right)$ is a given function, and $\emptyset \neq U_{a d}^{\varepsilon} \subset L^{2}\left(\mathbb{R}^{n}\right)$ is a convex and closed subset representing the admissible control set. By a classical argument $[30,33]$, the optimal control problem $\left(\mathrm{P}_{\varepsilon}\right)$ admits a unique optimal solution denoted throughout this paper by $\left(u_{\varepsilon}^{*}, y_{\varepsilon}^{*}\right) \in U_{a d}^{\varepsilon} \times H^{1}\left(\mathbb{R}^{n}\right)$. Let us emphasize that the admissible control set $U_{a d}^{\varepsilon}$ and the quantities $f^{\varepsilon}, y_{d, 1}^{\varepsilon}, y_{d, 2}^{\varepsilon}$ feature highly oscillatory structures depending on $\epsilon$. In particular, the numerical treatment of $\left(\mathrm{P}_{\varepsilon}\right)$ is extremely costly since the fine scale, represented by the small parameter $\varepsilon>0$, has to be resolved. The underlying $\varepsilon$-dependence in $\left(\mathrm{P}_{\varepsilon}\right)$ will be specified in Assumptions 1.8 and 1.10 .

More than two decades ago, Kesavan and Saint Jean Paulin [21] analyzed the effective behavior of a similar linear-quadratic elliptic optimal control problem. They disregarded the role of the desired state and specified the desired state in the energy norm to be zero, i.e., $\mu_{2}=0$ and $y_{d, 1}^{\varepsilon} \equiv 0$ in (1.2). However, differently from 
$\left(\mathrm{P}_{\varepsilon}\right)$, they allowed for a periodic coefficient $B^{\varepsilon}$ in the cost functional that may differ from the coefficient $A^{\varepsilon}$ in the state equation. The main contribution of [21] is the weak convergence for the optimal solution as $\epsilon \rightarrow 0$ towards the solution of an effective optimal control problem with an effective state equation. The effective coefficient $A^{\text {eff }}$ in the effective state equation corresponds to the classical effective coefficient from the periodic homogenization theory, while the effective coefficient $B^{\text {eff }}$ in the energy functional is a perturbation of the classical H-limit of $B^{\varepsilon}$. In the case $A^{\varepsilon}=B^{\varepsilon}$, that is relevant for us, one obtains $A^{\text {eff }}=B^{\text {eff }}$. Unfortunately, since the convergence property holds solely in the weak topology, the approximation of the effective optimal control problem could be far from precise. Indeed, from the classical homogenization theory, it is well known that solutions to highly oscillatory problems exhibit highly oscillatory behavior, and the homogenization limit provides an appropriate average of these oscillations. In order to obtain higher-order approximations, one has to capture the fast oscillations by correcting the homogenization limit, wherefore results of this type are referred to as corrector results. The ultimate goal of this paper is therefore to set up a corrector result of the following form: Given $M \in \mathbb{N}$, we seek for a proper approximation of the optimal solution $\left(u_{\varepsilon}^{*}, y_{\varepsilon}^{*}\right)$ to $\left(\mathrm{P}_{\varepsilon}\right)$ satisfying the a priori estimate (1.30) with error $O\left(\varepsilon^{2 M}\right)$. The key tool of our approach is the Bloch wave expansion (see Sect. 1.1) serving as the fundament of the higher-order effective optimal control problem $\left(\mathrm{P}_{M}^{*}\right)$ (see Def. 1.15) we focus on in this paper. Based on the proposed effective model $\left(\mathrm{P}_{M}^{*}\right)$, we are able to prove the desired corrector result.

The rest of of the paper is organized as follows. In the upcoming section, we recall the basics of the Bloch wave expansion. In Section 1.2, we present the assumptions for the data involved in the optimal control problem $\left(\mathrm{P}_{\varepsilon}\right)$. Section 1.3 formulates the effective optimal control problem $\left(\mathrm{P}_{M}^{*}\right)$ and summarizes the main result of the paper. The existence analysis of the effective model $\left(\mathrm{P}_{M}^{*}\right)$ is performed in Section 2. Thereafter, the desired approximation property of $\left(\mathrm{P}_{M}^{*}\right)$ is established in Section 3. The final section is devoted to the analysis of an alternative effective problem.

\subsection{Bloch expansion}

For the sake of completeness, we briefly motivate and collect well-known facts from the classical Bloch theory in the context of homogenization, which can be found, for instance, in $[13,14,17,32]$.

The Bloch expansion is a generalization of the classical Fourier expansion. Every function $v \in L^{2}\left(\mathbb{R}^{n} ; \mathbb{C}\right)$ can be written as

$$
v(x)=\int_{\mathbb{R}^{n}} \hat{v}(\xi) e^{2 \pi i \xi \cdot x} \mathrm{~d} \xi
$$

where $\hat{v}(\xi):=\int_{\mathbb{R}^{n}} v(x) e^{-2 \pi i x \cdot \xi} \mathrm{d} x$ denotes the classical Fourier-transform of $v$. Decomposing every $\xi \in \mathbb{R}^{n}$ into

$$
\xi=k+\eta \quad \text { with } k \in \mathbb{Z}^{n} \text { and } \eta \in Z:=[-1 / 2,1 / 2)^{n},
$$

one obtains

$$
v(x)=\int_{Z} \underbrace{\sum_{k \in \mathbb{Z}^{n}} \hat{v}(k+\eta) e^{2 \pi i k \cdot x}}_{=: F=F(x ; \eta)} e^{2 \pi i \eta \cdot x} \mathrm{~d} \eta,
$$

where for every $\eta \in Z$ the function $F(\cdot, \eta)$ is $Y$-periodic with $Y:=[0,1]^{n}$. It can thus be expanded in the $Y$-periodic eigenfunctions to the following eigenvalue problem:

Definition 1.1 (Bloch eigenvalue problem). Let $\eta \in Z=\left[-\frac{1}{2}, \frac{1}{2}\right)^{n}$ be fixed. Consider the operator

$$
\mathcal{L}(\eta):=-(\nabla+2 \pi i \eta) \cdot(A(\cdot)(\nabla+2 \pi i \eta)) .
$$


By the classical spectral theory, one finds a sequence of eigenvalues $\left(\mu_{m}(\eta)\right)_{m \in \mathbb{N}_{0}}$ and corresponding $Y$-periodic eigenfunctions $\left(\Phi_{m}(\cdot ; \eta)\right)_{m \in \mathbb{N}_{0}} \subset H_{\sharp}^{1}(Y ; \mathbb{C})$ of $\mathcal{L}(\eta)$, such that

$$
\begin{aligned}
& 0 \leq \lambda_{0}(\eta) \leq \lambda_{1}(\eta) \leq \ldots \text { with } \lambda_{m}(\eta) \rightarrow \infty \text { as } m \rightarrow \infty \quad \text { (Bloch eigenvalues), } \\
& \left(\Phi_{m}(\cdot ; \eta)\right)_{m \in \mathbb{N}_{0}} \text { form a ONB of } L_{\sharp}^{2}(Z ; \mathbb{C}) \quad \text { (Bloch waves). }
\end{aligned}
$$

The subscript $\sharp$ tags spaces of periodic functions. Furthermore we define

$$
\omega_{m}(x ; \eta):=\Phi_{m}(x ; \eta) e^{2 \pi i \eta \cdot x} \quad \text { (quasi-periodic Bloch waves). }
$$

Note that the quasi-periodic Bloch waves solve the eigenvalue problem

$$
-\nabla \cdot\left(A(x) \nabla \omega_{m}(x ; \eta)\right)=\lambda_{m}(\eta) \omega_{m}(x ; \eta) .
$$

This leads to the following classical result.

Proposition 1.2 (Bloch expansion). Every $v \in L^{2}\left(\mathbb{R}^{n} ; \mathbb{C}\right)$ admits a unique expansion

$$
v(x)=\int_{Z} \sum_{m \in \mathbb{N}_{0}} \hat{v}_{m}(\eta) \Phi_{m}(x ; \eta) e^{2 \pi i \eta \cdot x} \mathrm{~d} \eta=\int_{Z} \sum_{m \in \mathbb{N}_{0}} \hat{v}_{m}(\eta) \omega_{m}(x ; \eta) \mathrm{d} \eta
$$

with the Bloch coefficients

$$
\hat{v}_{m}(\eta):=\int_{\mathbb{R}^{n}} v(x) e^{-2 \pi i \eta \cdot x} \overline{\Phi_{m}(x ; \eta)} \mathrm{d} x \quad \text { for all } m \in \mathbb{N}_{0} \quad \text { and } \eta \in Z .
$$

Furthermore Parseval's identity holds

$$
\int_{\mathbb{R}^{n}}|v(x)|^{2} \mathrm{~d} x=\int_{Z} \sum_{m \in \mathbb{N}_{0}}\left|\hat{v}_{m}(\eta)\right|^{2} \mathrm{~d} \eta
$$

Since the coefficient $A^{\varepsilon}(x)=A\left(\frac{x}{\varepsilon}\right)$ in $\left(\mathrm{P}_{\varepsilon}\right)$ is $\varepsilon$-periodic, one needs to introduce rescaled Bloch waves:

$$
\begin{aligned}
& \Phi_{m}^{\varepsilon}(x ; \eta):=\Phi_{m}\left(\frac{x}{\varepsilon} ; \varepsilon \eta\right), \quad \lambda_{m}^{\varepsilon}(\eta):=\frac{1}{\varepsilon^{2}} \lambda_{m}(\varepsilon \eta), \\
& \omega_{m}^{\varepsilon}(x, \eta):=\omega_{m}\left(\frac{x}{\varepsilon} ; \varepsilon \eta\right)=\Phi_{m}^{\varepsilon}(x ; \eta) e^{2 \pi i \eta \cdot x}=\Phi_{m}\left(\frac{x}{\varepsilon} ; \varepsilon \eta\right) e^{2 \pi i \eta \cdot x} .
\end{aligned}
$$

The scaling is designed in such a way that

$$
-\nabla \cdot\left(A^{\varepsilon}(x) \nabla \omega_{m}^{\varepsilon}(x ; \eta)\right)=\lambda_{m}^{\varepsilon}(\eta) \omega_{m}^{\varepsilon}(x ; \eta) .
$$

A simple calculation provides the following rescaled version of the classical Bloch expansion.

Proposition 1.3 (Rescaled Bloch expansion). Every $v \in L^{2}\left(\mathbb{R}^{n} ; \mathbb{C}\right)$ admits a unique expansion

$$
\begin{aligned}
v(x) & =\int_{Z / \varepsilon} \sum_{m \in \mathbb{N}_{0}} \hat{v}_{m}^{\varepsilon}(\eta) \omega_{m}^{\varepsilon}(x ; \eta) \mathrm{d} \eta \\
\hat{v}_{m}^{\varepsilon}(\eta) & =\int_{\mathbb{R}^{n}} v(x) e^{-2 \pi i \eta \cdot x} \overline{\Phi_{m}^{\varepsilon}(x ; \eta)} \mathrm{d} x=\int_{\mathbb{R}^{n}} v(x) \overline{\omega_{m}^{\varepsilon}(x ; \eta)} \mathrm{d} x .
\end{aligned}
$$


Furthermore Parseval's identity holds

$$
\int_{\mathbb{R}^{n}}|v(x)|^{2} \mathrm{~d} x=\int_{Z / \varepsilon} \sum_{m \in \mathbb{N}_{0}}\left|\hat{v}_{m}^{\varepsilon}(\eta)\right|^{2} \mathrm{~d} \eta .
$$

In what follows, we will frequently exploit that due to the eigenvalue property of $\omega_{m}^{\varepsilon}$ the operator $-\nabla \cdot\left(A^{\varepsilon} \nabla\right)$ acts on the rescaled Bloch expansion as a multiplier. More precisely, for $v$ as in (1.9) one has

$$
-\nabla \cdot\left(A^{\varepsilon}(x) \nabla v(x)\right)+v(x)=\int_{Z / \varepsilon} \sum_{m \in \mathbb{N}_{0}}\left(1+\lambda_{m}^{\varepsilon}(\eta)\right) \hat{v}_{m}^{\varepsilon}(\eta) \omega_{m}^{\varepsilon}(x ; \eta) \mathrm{d} \eta
$$

provided $-\nabla \cdot\left(A^{\varepsilon} \nabla v\right) \in L^{2}\left(\mathbb{R}^{n}\right)$.

It is well known that in the homogenization process only the lowest eigenvalue, the ground state $\lambda_{0}(\eta)$, is relevant. By classical perturbation theory it can be shown that $\lambda_{0}(\eta)$ is simple and analytic in a neighbourhood of $\eta=0$. Moreover, one immediately finds that $\lambda_{0}(0)=0$ and that $\lambda_{0}$ is even, wherefore all odd derivatives of $\lambda_{0}$ vanish. Summing up, one obtains the following Taylor expansion:

Lemma 1.4 (Taylor expansion of the first Bloch eigenvalue). For every $k \in \mathbb{N}$ there exists a $2 k$ th order tensor $\mathbb{A}_{2 k}^{*}=\left(\mathbb{A}_{2 k}^{*}\right)_{i_{1}, \ldots, i_{2 k}}$ such that for $M \in \mathbb{N}$

$$
\lambda_{0}(\eta)=(2 \pi)^{2} \mathbb{A}_{2}^{*} \cdot \eta^{\otimes 2}+(2 \pi)^{4} \mathbb{A}_{4}^{*} \cdot \eta^{\otimes 4}+\cdots+(2 \pi)^{2 M} \mathbb{A}_{2 M}^{*} \cdot \eta^{\otimes 2 M}+O\left(|\eta|^{2 M+2}\right),
$$

where

$$
\mathbb{A}_{2 k}^{*} \cdot \eta^{\otimes 2 k}:=\sum_{i_{1}, \ldots, i_{2 k}=1}^{n}\left(\mathbb{A}_{2 k}^{*}\right)_{i_{1}, \ldots, i_{2 k}} \eta_{i_{1}} \cdots \cdots \eta_{i_{2 k}} .
$$

It is a well-known fact in the Bloch theory that the second order tensor $\mathbb{A}_{2}^{*} \in \mathbb{R}^{n \times n}$ coincides with the effective coefficient $A^{\text {eff }}$ from the classical homogenization theory. As shown, for instance in $[4,18]$, the tensors $\mathbb{A}_{2 k}^{*}$ can be computed from periodic cell problems. The above expansion plays a major role in our analysis.

\subsection{Assumptions}

In this section, we introduce the assumptions for the given data involved in $\left(\mathrm{P}_{\varepsilon}\right)$, namely, the admissible control set $U_{a d}^{\varepsilon} \subset L^{2}\left(\mathbb{R}^{n}\right)$ and the functions $f^{\varepsilon}, y_{d, 1}^{\varepsilon}$, and $y_{d, 2}^{\varepsilon}$. Roughly speaking, we have to demand that the data are well prepared to the periodic microstructure.

We recall that solutions to homogenization problems are characterized by some effective profile, the homogenization limit, and fast lower order oscillations, which adapt the effective profile to the periodic microstructure. These oscillatory corrections can be described by periodic cell solutions, the so-called correctors, which are necessary to construct high-order approximations. In fact, there is an analogue to this adaption process in the Bloch wave approach. As shown in [13, 14, 17], in a classical homogenization process only the lowest Bloch eigenvalue $\lambda_{0}^{\varepsilon}$ and the corresponding eigenfunction $\omega_{0}^{\varepsilon}$ play a role, while higher Bloch modes are negligible. Moreover, it can be shown that the rescaled Bloch eigenfunction $\omega_{0}^{\varepsilon}$ is in fact an adaption of the Fourier basis function $e^{2 \pi i \eta \cdot x}$ to the microstructure (see [4]). In view of this, the following definition is reasonable.

Definition 1.5 (Adaption). Let $f \in L^{2}\left(\mathbb{R}^{n} ; \mathbb{C}\right)$ with the Fourier-transform $\hat{f}$. Then, the adaption $\mathcal{A}^{\varepsilon}(f)$ of $f$ is defined as follows:

$$
\mathcal{A}^{\varepsilon}(f):=\int_{Z / \varepsilon} \hat{f}(\eta) \omega_{0}^{\varepsilon}(\cdot ; \eta) \mathrm{d} \eta
$$


Remark 1.6. In [14] $\mathcal{A}^{\varepsilon}$ is referred to as the Bloch approximation. It is shown that $\mathcal{A}^{\varepsilon}(f)$ converges almost everywhere to $f$ and, assuming that $f$ is sufficiently smooth with $\hat{f}$ decaying sufficiently fast at infinity, a higher-order asymptotic expansion of $\mathcal{A}^{\varepsilon}(f)$ holds

$$
\mathcal{A}^{\varepsilon}(f)(x)=f(x)+\varepsilon \chi^{(1)}\left(\frac{x}{\varepsilon}\right) \cdot \nabla f(x)+\varepsilon^{2} \chi^{(2)}\left(\frac{x}{\varepsilon}\right) \cdot D^{2} f(x)+O\left(\varepsilon^{3}\right),
$$

where $\chi^{(1)}, \chi^{(2)}$ are $Y$-periodic corrector functions. In particular, the adaption $\mathcal{A}^{\varepsilon}$ transforms a smooth, nonoscillatory function $f$ to an $\varepsilon$-oscillatory function according to the periodic microstructure.

In our approach we will use the fact that the adaption operator $\mathcal{A}^{\varepsilon}$ preserves the $L^{2}$-norm when applied to functions that have compact support in Fourier space.

Lemma 1.7. Let $f \in L^{2}\left(\mathbb{R}^{n} ; \mathbb{C}\right)$ have compact support in Fourier space, i.e., $\operatorname{supp}(\hat{f}) \subset K$ with $K \subset \mathbb{R}^{n}$ compact. Let $\varepsilon>0$ be such that $K \subset Z / \varepsilon$. Then

$$
\left\|\mathcal{A}^{\varepsilon}(f)\right\|_{L^{2}\left(\mathbb{R}^{n} ; \mathbb{C}\right)}=\|f\|_{L^{2}\left(\mathbb{R}^{n} ; \mathbb{C}\right)} .
$$

Proof. Let $\varepsilon>0$ be such that $K \subset Z / \varepsilon$. Then

$$
\|f\|_{L^{2}\left(\mathbb{R}^{n} ; \mathbb{C}\right)}^{2}=\int_{\mathbb{R}^{n}}|\hat{f}(\eta)|^{2} \mathrm{~d} \eta=\int_{Z / \varepsilon}|\hat{f}(\eta)|^{2} \mathrm{~d} \eta=\left\|\mathcal{A}^{\varepsilon}(f)\right\|_{L^{2}\left(\mathbb{R}^{n} ; \mathbb{C}\right)}^{2},
$$

where in the second equality we exploited that $\hat{f}$ is supported on $K \subset Z / \varepsilon$ and in the last equality we used Parseval's identity (1.11) and the definition of $\mathcal{A}^{\varepsilon}$.

Let us now state the main assumptions for the given data and the admissible set $U_{a d}^{\varepsilon}$ involved in $\left(\mathrm{P}_{\varepsilon}\right)$ :

Assumption 1.8. There exist $f, y_{d, 1}, y_{d, 2} \in L^{2}\left(\mathbb{R}^{n}\right)$ and a compact set $K \subset \mathbb{R}^{n}$ such that

$$
\begin{aligned}
& f^{\varepsilon}=\mathcal{A}^{\varepsilon}(f), \quad y_{d, 1}^{\varepsilon}=\mathcal{A}^{\varepsilon}\left(y_{d, 1}\right) \quad \text { and } y_{d, 2}^{\varepsilon}=\mathcal{A}^{\varepsilon}\left(y_{d, 2}\right), \\
& \operatorname{supp}(\hat{f}), \operatorname{supp}\left(\hat{y}_{d, 1}\right), \operatorname{supp}\left(\hat{y}_{d, 2}\right) \subset K .
\end{aligned}
$$

Moreover, the compact set $K$ is assumed to be symmetric in the sense that

$$
\eta \in K \Rightarrow-\eta \in K
$$

\section{Remark 1.9.}

(i) The condition that the Fourier transforms $\hat{f}, \hat{y}_{d, 1}, \hat{y}_{d, 2}$ have compact support implies readily that $f, y_{d, 1}, y_{d, 2} \in H^{k}\left(\mathbb{R}^{n}\right)$ for all $k \in \mathbb{N}$. Therefore, along with (1.16), it follows that their adaptions $f^{\varepsilon}, y_{d, 1}^{\varepsilon}, y_{d, 2}^{\varepsilon}$ are real-valued and belong to $H^{1}\left(\mathbb{R}^{n}\right), c f$. [13,14]. Also, notice that Assumption 1.8 demands that $f^{\varepsilon}, y_{d, 1}^{\varepsilon}$ and $y_{d, 2}^{\varepsilon}$ are highly oscillatory according to the micro-structure. Actually this assumption is quite natural as solutions to $\varepsilon$-periodic coefficient equations such as the state equation in $\left(\mathrm{P}_{\varepsilon}\right)$ typically exhibit a highly oscillatory behavior. We note that the assumption on the source term $f$ having a compact support in Fourier space is rather restrictive. It would be more natural to consider sources $f$ in Schwartz space. The strong decay would still be sufficient to exploit the analyticity of the lowest Bloch eigenvalue and to derive high order estimates. We shall consider this case in our future work based on Schwartz space properties.

(ii) Relation (1.14) suggests that the difference $\mathcal{A}^{\varepsilon}(f)-f$ is typically of order $\varepsilon$. For unprepared data, i.e., $y_{d, 1}^{\varepsilon}=y_{d, 1}, y_{d, 2}^{\varepsilon}=y_{d, 2}$, and $f^{\varepsilon}=f$, the effective model from Definition 1.15 below is therefore expected to approximate the original problem only up to errors of order $\varepsilon$. In fact, well-prepared data are necessary for 
many corrector results in the homogenization theory. See, for instance, $[5,7,10]$ in the context of periodic wave equations.

We now state the assumption on the admissible set $U_{a d}^{\varepsilon}$.

Assumption 1.10. Let $K \subset \mathbb{R}^{n}$ be as in Assumption 1.8. For the admissible set $U_{a d}^{\varepsilon} \subset L^{2}\left(\mathbb{R}^{n}\right)$, we assume the following:

(i) $U_{a d}^{\varepsilon}$ is convex and closed.

(ii) There exists $u_{0} \in L^{2}\left(\mathbb{R}^{n}\right)$ such that for every $\varepsilon>0$ one has $\mathcal{A}^{\varepsilon}\left(u_{0}\right) \in U_{a d}^{\varepsilon}$.

(iii) For every $u \in U_{a d}^{\varepsilon}$ with Bloch expansion

$$
u(x)=\int_{Z / \varepsilon} \sum_{m \in \mathbb{N}_{0}} \hat{u}_{m}^{\varepsilon}(\eta) \omega_{m}^{\varepsilon}(x ; \eta) \mathrm{d} \eta,
$$

it follows that the projection

$$
u_{K}(x):=\int_{K} \hat{u}_{0}^{\varepsilon}(\eta) \omega_{0}^{\varepsilon}(x ; \eta) \mathrm{d} \eta
$$

lies as well in $U_{a d}^{\varepsilon}$. In other words, $\left(U_{a d}^{\varepsilon}\right)_{K}:=\left\{u_{K} \mid u \in U_{a d}^{\varepsilon}\right\} \subset U_{a d}^{\varepsilon}$.

Remark 1.11. The second condition (ii) guarantees that $\left(u_{\varepsilon}^{*}\right)_{\epsilon>0} \subset L^{2}\left(\mathbb{R}^{n}\right)$ is uniformly bounded as shown in Lemma 1.13. The third condition (iii) demands that for every $\varepsilon>0$ the projection $\left(U_{a d}^{\varepsilon}\right)_{K}$ of $U_{a d}^{\varepsilon}$ in Bloch space to the lowest mode $m=0$ and $\eta \in K$ is a subset of $U_{a d}^{\varepsilon}$.

Remark 1.12. It should be underlined that the assumption on compact support in Fourier space is solely considered for the data $f, y_{d, 1}$, and $y_{d, 2}$ (see Assumption 1.8). We do not impose such a compact assumption on the admissible control set $U_{a d}^{\varepsilon}$ (see Assm. 1.10). Later on, our analysis (Prop. 2.2) eventually reveals that the optimal control $u_{\varepsilon}^{*}$ of $\left(\mathrm{P}_{\varepsilon}\right)$ turns out to be the adaption of some function with compact support $K$ in Fourier space.

For the rest of the article, $\varepsilon>0$ is always assumed to be sufficiently small such that $K \subset Z / \varepsilon$. Furthermore $S_{\epsilon}: L^{2}\left(\mathbb{R}^{n}\right) \rightarrow H^{1}\left(\mathbb{R}^{n}\right)$ denotes the control-to-state operator associated with $\left(\mathrm{P}_{\varepsilon}\right)$.

Lemma 1.13. Let Assumptions 1.8 and 1.10 hold. Then, $\left(u_{\varepsilon}^{*}\right)_{\varepsilon>0} \subset L^{2}\left(\mathbb{R}^{n}\right)$ is uniformly bounded, i.e., there exists a constant $C>0$, independent of $\varepsilon$, such that

$$
\left\|u_{\varepsilon}^{*}\right\|_{L^{2}\left(\mathbb{R}^{n}\right)} \leq C \quad \forall \epsilon>0 .
$$

Proof. According to Assumption 1.10, there exists an element $u_{0} \in L^{2}\left(\mathbb{R}^{n}\right)$ such that $\mathcal{A}^{\varepsilon}\left(u_{0}\right) \in U_{a d}^{\varepsilon}$. For this reason,

$$
\frac{\kappa}{2}\left\|u_{\varepsilon}^{*}\right\|_{L^{2}\left(\mathbb{R}^{n}\right)}^{2} \leq J^{\varepsilon}\left(u_{\varepsilon}^{*}, S_{\epsilon} u_{\varepsilon}^{*}\right) \leq J^{\varepsilon}(\mathcal{A}^{\varepsilon}\left(u_{0}\right), \underbrace{S_{\epsilon} \mathcal{A}^{\varepsilon}\left(u_{0}\right)}_{=: y_{0}^{\varepsilon}}) \quad \forall \epsilon>0 .
$$

Our aim is to find a uniform bound for the right hand side of (1.18).

Let $\hat{u}_{0}$ be the Fourier transform of $u_{0}$. By construction of $\mathcal{A}^{\varepsilon}$ (Def. 1.5), Proposition 1.3 yields that the lowest-order Bloch-coefficient $(m=0)$ of $\mathcal{A}^{\varepsilon}\left(u_{0}\right)$ corresponds to the Fourier transform $\hat{u}_{0}$, while all higher-order Bloch modes with $m \geq 1$ vanish. In the following, let $C>0$ denote a $\varepsilon$-independent generic constant that can vary from line to line. By Lemma 2.1 below, the right-hand side in (1.18) can be written as

$$
J^{\varepsilon}\left(\mathcal{A}^{\varepsilon}\left(u_{0}\right), y_{0}^{\varepsilon}\right)=\frac{\mu_{1}}{2} \int_{K}\left|\frac{\hat{f}(\eta)+\hat{u}_{0}(\eta)}{1+\lambda_{0}^{\varepsilon}(\eta)}-\hat{y}_{d, 1}(\eta)\right|^{2}\left(1+\lambda_{0}^{\varepsilon}(\eta)\right) \mathrm{d} \eta+\frac{\mu_{1}}{2} \int_{(Z / \varepsilon) \backslash K} \frac{\left|\hat{u}_{0}(\eta)\right|^{2}}{1+\lambda_{0}^{\varepsilon}(\eta)} \mathrm{d} \eta
$$




$$
\begin{array}{r}
+\frac{\mu_{2}}{2} \int_{K}\left|\frac{\hat{f}(\eta)+\hat{u}_{0}(\eta)}{1+\lambda_{0}^{\varepsilon}(\eta)}-\hat{y}_{d, 2}(\eta)\right|^{2} \mathrm{~d} \eta+\frac{\mu_{2}}{2} \int_{(Z / \varepsilon) \backslash K} \frac{\left|\hat{u}_{0}(\eta)\right|^{2}}{\left(1+\lambda_{0}^{\varepsilon}(\eta)\right)^{2}} \mathrm{~d} \eta+\frac{\kappa}{2} \int_{Z / \varepsilon}\left|\hat{u}_{0}(\eta)\right|^{2} \mathrm{~d} \eta, \\
\leq C\left(\int_{Z / \varepsilon}\left|\hat{u}_{0}(\eta)\right|^{2} \mathrm{~d} \eta+\int_{K}|\hat{f}(\eta)|^{2}+\left|\hat{y}_{d, 1}(\eta)\right|^{2}\left(1+\lambda_{0}^{\varepsilon}(\eta)\right)+\left|\hat{y}_{d, 2}(\eta)\right|^{2} \mathrm{~d} \eta\right) \\
\leq C\left(\left\|u_{0}\right\|_{L^{2}\left(\mathbb{R}^{n}\right)}^{2}+\|f\|_{L^{2}\left(\mathbb{R}^{n}\right)}^{2}+\left\|y_{d, 1}\right\|_{H^{1}\left(\mathbb{R}^{n}\right)}^{2}+\left\|y_{d, 2}\right\|_{L^{2}\left(\mathbb{R}^{n}\right)}^{2}\right),
\end{array}
$$

where in the first inequality we used that $\lambda_{0}^{\varepsilon} \geq 0$. In the second inequality we used Parseval's identity (1.11) and exploited the fact that $\int_{K}\left|\hat{y}_{d, 1}(\eta)\right|^{2}\left(1+\lambda_{0}^{\varepsilon}(\eta)\right) \mathrm{d} \eta$ is controlled by the $H^{1}$-norm of $y_{d, 1}$ (see [14] for details).

Example 1.14. Let Assumption 1.8 hold. Then the following admissible control sets satisfy Assumption 1.10.

(i) Full space $U_{a d}^{\varepsilon}=L^{2}\left(\mathbb{R}^{n}\right)$.

(ii) Bounds on the $L^{2}$-norm of the control: For $L>0$ let

$$
U_{a d}^{\varepsilon}:=\left\{u \in L^{2}\left(\mathbb{R}^{n}\right) \mid\|u\|_{L^{2}\left(\mathbb{R}^{n}\right)} \leq L\right\} .
$$

It is obvious that $U_{a d}^{\varepsilon} \subset L^{2}\left(\mathbb{R}^{n}\right)$ is convex and closed. The condition (ii) of Assumption 1.10 is satisfied with $u_{0}=0$. The third condition is a direct consequence of (1.17) and Parseval's identity (1.11).

(iii) Bounds on the $L^{2}$-norm of the state: For $L>0$ let

$$
U_{a d}^{\varepsilon}:=\left\{u \in L^{2}\left(\mathbb{R}^{n}\right) \mid\left\|S_{\epsilon} u\right\|_{L^{2}\left(\mathbb{R}^{n}\right)} \leq L\right\} .
$$

As the control-to-state operator $S_{\epsilon}: L^{2}\left(\mathbb{R}^{n}\right) \rightarrow H^{1}\left(\mathbb{R}^{n}\right)$ is affine linear and continuous, the set $U_{a d}^{\varepsilon} \subset$ $L^{2}\left(\mathbb{R}^{n}\right)$ is convex and closed. The condition (ii) of Assumption 1.10 is satisfied with $u_{0}=-f$ since according to Assumption 1.8

$$
S_{\epsilon}\left(\mathcal{A}^{\varepsilon}(-f)\right)=S_{\epsilon}\left(-f^{\varepsilon}\right)=0 \quad \Rightarrow \quad \mathcal{A}^{\varepsilon}(-f) \in U_{a d}^{\varepsilon} \quad \forall \epsilon>0 .
$$

The third condition of Assumption 1.10 follows from the formula (2.1) and Parseval's identity (1.11) implying $\left\|S_{\epsilon} u_{K}\right\|_{L^{2}\left(\mathbb{R}^{n}\right)} \leq\left\|S_{\epsilon} u\right\|_{L^{2}\left(\mathbb{R}^{n}\right)} \leq L$ for all $u \in U_{a d}$.

(iv) Bounds on the energy of the state: For $L>0$ let

$$
U_{a d}^{\varepsilon}:=\left\{u \in L^{2}\left(\mathbb{R}^{n}\right) \mid E^{\varepsilon}\left(S_{\epsilon} u\right) \leq L\right\} .
$$

Since by (1.1) the energy functional $E^{\varepsilon}: H^{1}\left(\mathbb{R}^{n}\right) \rightarrow \mathbb{R}$ defines a norm on $H^{1}\left(\mathbb{R}^{n}\right)$, the set $U_{a d}^{\varepsilon} \subset L^{2}\left(\mathbb{R}^{n}\right)$ is again convex and closed. Thanks to (1.19), the choice $u_{0}=-f$ satisfies again the condition (ii) of Assumption 1.10. In view of $(2.2)$ with $\hat{y}_{d, 1} \equiv 0$, the third condition $\left(U_{a d}^{\varepsilon}\right)_{K} \subset U_{a d}^{\varepsilon}$ is again satisfied by Parseval's identity (1.11).

(v) Adaption of a fixed set: Suppose that $U \subset L^{2}\left(\mathbb{R}^{n}\right)$ is a convex and closed subset such that

$$
U_{a d}:=\left\{u \in U \mid u(x)=\int_{K} \hat{u}(\eta) e^{2 \pi i \eta \cdot x} \mathrm{~d} \eta\right\}
$$

is nonempty. We consider

$$
U_{a d}^{\varepsilon}:=\mathcal{A}^{\varepsilon}\left(U_{a d}\right) .
$$

In view of (1.20), it holds that $U_{a d}^{\varepsilon}=\mathcal{A}^{\varepsilon}\left(U_{a d}\right)=\left(U_{a d}^{\varepsilon}\right)_{K}$. Moreover, the condition (ii) of Assumption 1.10 is satisfied since $\mathcal{A}^{\varepsilon}(u) \in U_{a d}^{\varepsilon}$ holds for all $u \in U_{a d}$ and all $\epsilon>0$. The convexity of $U_{a d}^{\varepsilon}=\mathcal{A}^{\varepsilon}\left(U_{a d}\right) \subset$ 
$L^{2}\left(\mathbb{R}^{n}\right)$ follows immediately from the linearity of the adaption $\mathcal{A}^{\varepsilon}$ and the convexity of $U$ and thus of $U_{a d} \subset L^{2}\left(\mathbb{R}^{n}\right)$. To prove the closedness, let $\left\{y_{j}\right\}_{j=1}^{\infty} \subset U_{a d}^{\varepsilon}$ be a sequence converging strongly in $L^{2}\left(\mathbb{R}^{n}\right)$ towards $y \in L^{2}\left(\mathbb{R}^{n}\right)$, i.e.,

$$
0=\lim _{j \rightarrow \infty} \int_{\mathbb{R}^{n}}\left|y_{j}(x)-y(x)\right|^{2} \mathrm{~d} x \underbrace{=}_{(1.11)} \lim _{j \rightarrow \infty} \int_{Z / \varepsilon} \sum_{m \in \mathbb{N}_{0}}\left|\hat{y}_{j, m}^{\varepsilon}(\eta)-\hat{y}_{m}^{\varepsilon}(\eta)\right|^{2} \mathrm{~d} \eta,
$$

where $\hat{y}_{j, m}^{\varepsilon}$ (resp. $\hat{y}_{m}^{\varepsilon}$ ) denote the Bloch-coefficients associated with $y_{j}$ (resp. $y$ ) for all $j \in \mathbb{N}$. As $U_{a d}^{\varepsilon}=$ $\mathcal{A}^{\varepsilon}\left(U_{a d}\right)$, we find a sequence $\left\{u_{j}\right\}_{j=1}^{\infty} \subset U_{a d}$ such that $y_{j}=\mathcal{A}^{\varepsilon}\left(u_{j}\right)$ for all $j \in \mathbb{N}$, from which it follows due to $(1.20)$ that

$$
\hat{y}_{j, 0}^{\varepsilon}=\left\{\begin{array}{lr}
\hat{u}_{j} & \text { in } K \\
0 & \text { in }(Z / \varepsilon) \backslash K
\end{array} \quad \text { and } \quad \hat{y}_{j, m}^{\varepsilon} \equiv 0 \quad \forall j, m \in \mathbb{N} .\right.
$$

Applying (1.23) to (1.22) yields

$$
y(\cdot)=\int_{K} \hat{y}_{0}^{\varepsilon}(\eta) \omega_{0}^{\varepsilon}(\cdot ; \eta) \mathrm{d} \eta \quad \text { and } \quad \lim _{j \rightarrow \infty} \int_{K}\left|\hat{u}_{j}(\eta)-\hat{y}_{0}^{\varepsilon}(\eta)\right|^{2} \mathrm{~d} \eta=0
$$

Introducing $u(x):=\int_{K} \hat{y}_{0}^{\varepsilon}(\eta) e^{2 \pi i \eta \cdot x} \mathrm{~d} \eta$, the above convergence in the Fourier space implies that $u_{j}$ converges strongly in $L^{2}\left(\mathbb{R}^{n}\right)$ towards $u$. Thus, as $U \subset L^{2}\left(\mathbb{R}^{n}\right)$ is closed, it follows by definition that $u \in U_{a d}$ and $y=\mathcal{A}^{\varepsilon}(u) \in U_{a d}^{\varepsilon}$.

Note that pointwise conditions on $u$ or $y$ are not covered by our approach. However, since solutions to homogenization problems typically exhibit a highly oscillatory behavior, pointwise conditions are not appropriate in the framework of high-order homogenization.

\subsection{Main results}

In the following we will expand the optimal solution $\left(u_{\varepsilon}^{*}, y_{\varepsilon}^{*}\right)$ of $\left(\mathrm{P}_{\varepsilon}\right)$ in rescaled Bloch waves according to Proposition 1.3. Our analysis consists of four steps: In the first step, see Proposition 2.2, we find that the optimal solution $\left(u_{\varepsilon}^{*}, y_{\varepsilon}^{*}\right)$ is in fact an adaption:

$$
u_{\varepsilon}^{*}=\mathcal{A}^{\varepsilon}\left(\tilde{u}_{\varepsilon}^{*}\right) \quad \text { and } \quad y_{\varepsilon}^{*}=\mathcal{A}^{\varepsilon}\left(\tilde{y}_{\varepsilon}^{*}\right)
$$

for some appropriate pair $\left(\tilde{u}_{\varepsilon}^{*}, \tilde{y}_{\varepsilon}^{*}\right)$. In the second step, we simplify the (Fourier) expansions of $\tilde{u}_{\varepsilon}^{*}$ and $\tilde{y}_{\varepsilon}^{*}$ using the analyticity of the lowest Bloch eigenvalue. Based on this first approximation, in our third step, we derive an $M$ th order effective optimal control problem of the following form.

Definition 1.15 (High-order effective optimal control problem). Let Assumptions 1.8 and 1.10 hold. Then, for every $M \in \mathbb{N}$, we define the high-order effective cost functional $J_{M}^{*}: L^{2}\left(\mathbb{R}^{n}\right) \times H^{M}\left(\mathbb{R}^{n}\right) \rightarrow \mathbb{R}$ as follows:

$$
\begin{gathered}
J_{M}^{*}(u, y):=\frac{\mu_{1}}{2} E_{M}^{*}\left(y-y_{d, 1}\right)+\frac{\mu_{2}}{2} \int_{\mathbb{R}^{n}}\left|y(x)-y_{d, 2}(x)\right|^{2} \mathrm{~d} x+\frac{\kappa}{2} \int_{\mathbb{R}^{n}}|u(x)|^{2} \mathrm{~d} x, \\
E_{M}^{*}(y):=\int_{\mathbb{R}^{n}}\left(\sum_{k=1}^{M} \varepsilon^{2 k-2}\left\langle D^{k} y(x), D^{k} y(x)\right\rangle_{\mathbb{A}_{2 k}^{*}}+|y(x)|^{2}\right) \mathrm{d} x
\end{gathered}
$$


where the tensors $\mathbb{A}_{2 k}^{*}$ are as in Lemma 1.4 and

$$
\left\langle D^{k} y(x), D^{k} y(x)\right\rangle_{\mathbb{A}_{2 k}^{*}}:=\sum_{i_{1}, \ldots, i_{2 k}=1}^{n}\left(\mathbb{A}_{2 k}^{*}\right)_{i_{1}, \ldots, i_{2 k}} \partial_{x_{i_{1}} x_{i_{2}} \ldots x_{i_{k}}}^{k} y(x) \partial_{x_{i_{k+1}} x_{i_{k}+2} \ldots x_{i_{2 k}}}^{k} y(x) .
$$

Introducing $\mathbb{A}_{2 k}^{*} D^{2 k}:=\sum_{i_{1}, \ldots, i_{2 k}=1}^{n}\left(\mathbb{A}_{2 k}^{*}\right)_{i_{1}, \ldots, i_{2 k}} \partial_{x_{i_{1}} x_{i_{2}} \ldots x_{i_{2 k}}}^{2 k}$, we consider the effective state equation

$$
\sum_{k=1}^{M} \varepsilon^{2 k-2}(-1)^{k} \mathbb{A}_{2 k}^{*} D^{2 k} y(x)+y(x)=f(x)+u(x)
$$

and propose the following high-order effective optimal control problem:

$$
\left\{\begin{aligned}
\min & J_{M}^{*}(u, y) \\
\text { over } & (u, y) \in U_{a d}^{*} \times H^{2 M}\left(\mathbb{R}^{n}\right) \\
\text { s.t. } & (1.27)
\end{aligned}\right.
$$

where the effective admissible control set $U_{a d}^{*}$ is given by the inverse image of $U_{a d}^{\varepsilon}$ under the adaption operator $\mathcal{A}^{\varepsilon}$ projected onto $K$,

$$
U_{a d}^{*}:=\left\{u(x)=\int_{K} \hat{u}(\eta) e^{2 \pi i \eta \cdot x} \mathrm{~d} \eta \mid \mathcal{A}^{\varepsilon}(u) \in U_{a d}^{\varepsilon}\right\} .
$$

In the fourth and final step, we show that $\left(\mathrm{P}_{M}^{*}\right)$ admits for all sufficiently small $\varepsilon>0$ a unique optimal solution $\left(u_{M}^{*}, y_{M}^{*}\right) \in U_{a d}^{*} \times H^{2 M}\left(\mathbb{R}^{n}\right)$, which relies on a reformulation of the problem in Fourier space (see Prop. 2.8). As a main result, we prove in Proposition 3.3 and Corollary 3.2 that the optimal solution $\left(u_{M}^{*}, y_{M}^{*}\right)$ to $\left(\mathrm{P}_{M}^{*}\right)$ satisfies the error estimate

$$
\left\|\tilde{u}_{\varepsilon}^{*}-u_{M}^{*}\right\|_{L^{2}\left(\mathbb{R}^{n}\right)}+\left\|\tilde{y}_{\varepsilon}^{*}-y_{M}^{*}\right\|_{L^{2}\left(\mathbb{R}^{n}\right)} \leq C \varepsilon^{2 M}
$$

for some $\varepsilon$-independent constant $C>0$. We recall that the optimal solution to $\left(\mathrm{P}_{\varepsilon}\right)$ satisfies $u_{\varepsilon}^{*}=\mathcal{A}^{\varepsilon}\left(\tilde{u}_{\varepsilon}^{*}\right)$ and $y_{\varepsilon}^{*}=\mathcal{A}^{\varepsilon}\left(\tilde{y}_{\varepsilon}^{*}\right)$. Since the functions $\tilde{u}_{\varepsilon}^{*}, u_{M}^{*}, \tilde{y}_{\varepsilon}^{*}$, and $y_{M}^{*}$ have compact support in Fourier space, Lemma 1.7 and (1.29) yield our final result:

Corollary 1.16. For all $\epsilon<\epsilon_{M}$ with $\epsilon_{M}$ provided by Lemma 2.5, it holds that

$$
\left\|u_{\varepsilon}^{*}-\mathcal{A}^{\varepsilon}\left(u_{M}^{*}\right)\right\|_{L^{2}\left(\mathbb{R}^{n}\right)}+\left\|y_{\varepsilon}^{*}-\mathcal{A}^{\varepsilon}\left(y_{M}^{*}\right)\right\|_{L^{2}\left(\mathbb{R}^{n}\right)} \leq C \varepsilon^{2 M} .
$$

Let us comment on the high-order effective optimal control problem $\left(\mathrm{P}_{M}^{*}\right)$. This optimization problem still depends on $\varepsilon$. It can nevertheless be regarded as an effective model in the sense of the homogenization theory since the coefficients $\mathbb{A}_{2 k}^{*}$ in the effective cost functional $J_{M}^{*}$ and the effective equation (1.27) are $\varepsilon$-independent. Note that, for $M=1,\left(\mathrm{P}_{M}^{*}\right)$ coincides with the classical homogenization limit obtained in [21]. For $M>1$ it can be understood as a higher-order approximation. The effective admissible set $U_{a d}^{*}$ may depend on $\varepsilon$. In Section 2.2 we determine $U_{a d}^{*}$ corresponding to the admissible sets from Example 1.14.

Remark 1.17. As shown in Section 2.2 below the effective state equation (1.27) is well-posed for $\varepsilon>0$ lying below a certain threshold $\varepsilon_{M}$. Such a threshold can only be found if the right hand side of (1.27) has compact support in Fourier space. This is why the effective admissible set (1.28) is restricted to functions with compact support $K$ in Fourier space. In Section 4 we provide an alternative $M$ th order effective optimal control problem which is well-posed independently of $\varepsilon$ and which has the same approximation quality as 
HIGH-ORDER HOMOGENIZATION IN OPTIMAL CONTROL

the one from Definition 1.15. In that situation the effective admissible set may be defined as $\left(\mathcal{A}^{\varepsilon}\right)^{-1}\left(U_{a d}^{\varepsilon}\right)=$ $\left\{u \in L^{2}\left(\mathbb{R}^{n}\right) \mid \mathcal{A}^{\varepsilon}(u)=\int_{Z / \epsilon} \hat{u}(\eta) \omega_{0}^{\epsilon}(x ; \eta) \mathrm{d} \eta \in U_{a d}^{\varepsilon}\right\}$. The difference to the admissible set $U_{a d}^{*}$ from (1.28) is that the Fourier transform of $u \in\left(\mathcal{A}^{\varepsilon}\right)^{-1}\left(U_{a d}^{\varepsilon}\right)$ is not restricted to the compact set $K$ thus allowing for much less regular controls. However, the effective optimal control still lies in the projected set $U_{a d}^{*}$.

In the following section, we derive and justify the effective model $\left(\mathrm{P}_{M}^{*}\right)$, and in Section 2.2 we show that it admits a unique optimal solution for all sufficiently small $\varepsilon>0$. Section 3 provides the central error estimates and proves the approximation property of $\left(\mathrm{P}_{M}^{*}\right)$. In this context a high-order approximation for the adjoint state is obtained. In Section 4 an alternative effective problem is proposed, which is well-posed independently of $\varepsilon$ and related to $\left(\mathrm{P}_{M}^{*}\right)$ through an algebraic manipulation.

\section{The EFFECTIVE OPTIMAL CONTROL PROBLEM}

In this section, we justify the $M$ th order effective optimal control problem $\left(\mathrm{P}_{M}^{*}\right)$. The key steps in the derivation are (1) rewriting the original problem in Bloch space and (2) expanding the lowest Bloch eigenvalue in a Taylor series (see Sect. 2.1). In Section 2.2 we prove that for all sufficiently small $\varepsilon>0,\left(\mathrm{P}_{M}^{*}\right)$ admits a unique optimal solution.

\subsection{Derivation of the effective model}

As the first step, using Proposition 1.3, we expand the optimal solution $\left(u_{\varepsilon}^{*}, y_{\varepsilon}^{*}\right)$ to $\left(\mathrm{P}_{\varepsilon}\right)$ in the rescaled Bloch waves as follows:

$$
u_{\varepsilon}^{*}(x)=\int_{Z / \varepsilon} \sum_{m \in \mathbb{N}_{0}} \hat{u}_{\varepsilon, m}^{*}(\eta) \omega_{m}^{\varepsilon}(x ; \eta) \mathrm{d} \eta \quad \text { and } \quad y_{\varepsilon}^{*}(x) \quad=\int_{Z / \varepsilon} \sum_{m \in \mathbb{N}_{0}} \hat{y}_{\varepsilon, m}^{*}(\eta) \omega_{m}^{\varepsilon}(x ; \eta) \mathrm{d} \eta .
$$

We then specify the Bloch expansion of the optimal state $y_{\varepsilon}^{*}(x)$ and rewrite the cost functional $J^{\varepsilon}$ in terms of the Bloch transform.

Lemma 2.1. Let Assumptions 1.8 and 1.10 hold. Then, for every control $u_{\varepsilon} \in U_{a d}^{\varepsilon}$ with Bloch coefficients $\hat{u}_{\varepsilon, m}$, the corresponding state $y_{\varepsilon}:=S_{\epsilon} u_{\epsilon}$ satisfies the following expansion:

$$
\begin{aligned}
y_{\varepsilon}(x)= & \int_{K} \frac{\hat{f}(\eta)+\hat{u}_{\varepsilon, 0}(\eta)}{1+\lambda_{0}^{\varepsilon}(\eta)} \omega_{0}^{\varepsilon}(x ; \eta) \mathrm{d} \eta+\int_{(Z / \varepsilon) \backslash K} \frac{\hat{u}_{\varepsilon, 0}(\eta)}{1+\lambda_{0}^{\varepsilon}(\eta)} \omega_{0}^{\varepsilon}(x ; \eta) \mathrm{d} \eta \\
& +\int_{Z / \varepsilon} \sum_{m \geq 1} \frac{\hat{u}_{\varepsilon, m}(\eta)}{1+\lambda_{m}^{\varepsilon}(\eta)} \omega_{m}^{\varepsilon}(x ; \eta) \mathrm{d} \eta,
\end{aligned}
$$

and the cost functional $J^{\varepsilon}$ is given by

$$
J^{\varepsilon}\left(u_{\varepsilon}, y_{\varepsilon}\right)=\hat{J}^{\varepsilon}\left(\hat{u}_{\varepsilon, m}\right):=\frac{\mu_{1}}{2} \hat{J}_{1}^{\varepsilon}\left(\hat{u}_{\varepsilon, m}\right)+\frac{\mu_{2}}{2} \hat{J}_{2}^{\varepsilon}\left(\hat{u}_{\varepsilon, m}\right)+\frac{\kappa}{2} \int_{Z / \varepsilon} \sum_{m \in \mathbb{N}_{0}}\left|\hat{u}_{\varepsilon, m}(\eta)\right|^{2} \mathrm{~d} \eta,
$$

where

$$
\begin{aligned}
\hat{J}_{1}^{\varepsilon}\left(\hat{u}_{\varepsilon, m}\right)= & \int_{K}\left|\frac{\hat{f}(\eta)+\hat{u}_{\varepsilon, 0}(\eta)}{1+\lambda_{0}^{\varepsilon}(\eta)}-\hat{y}_{d, 1}(\eta)\right|^{2}\left(1+\lambda_{0}^{\varepsilon}(\eta)\right) \mathrm{d} \eta+\int_{(Z / \varepsilon) \backslash K} \frac{\left|\hat{u}_{\varepsilon, 0}(\eta)\right|^{2}}{1+\lambda_{0}^{\varepsilon}(\eta)} \mathrm{d} \eta \\
& +\int_{Z / \varepsilon} \sum_{m \geq 1} \frac{\left|\hat{u}_{\varepsilon, m}(\eta)\right|^{2}}{1+\lambda_{m}^{\varepsilon}(\eta)} \mathrm{d} \eta,
\end{aligned}
$$




$$
\begin{aligned}
\hat{J}_{2}^{\varepsilon}\left(\hat{u}_{\varepsilon, m}\right)= & \int_{K}\left|\frac{\hat{f}(\eta)+\hat{u}_{\varepsilon, 0}(\eta)}{1+\lambda_{0}^{\varepsilon}(\eta)}-\hat{y}_{d, 2}(\eta)\right|^{2} \mathrm{~d} \eta+\int_{(Z / \varepsilon) \backslash K} \frac{\left|\hat{u}_{\varepsilon, 0}(\eta)\right|^{2}}{\left(1+\lambda_{0}^{\varepsilon}(\eta)\right)^{2}} \mathrm{~d} \eta \\
& +\int_{Z / \varepsilon} \sum_{m \geq 1} \frac{\left|\hat{u}_{\varepsilon, m}(\eta)\right|^{2}}{\left(1+\lambda_{m}^{\varepsilon}(\eta)\right)^{2}} \mathrm{~d} \eta .
\end{aligned}
$$

Proof. Thanks to (1.15), the Bloch coefficients of $f^{\varepsilon}=\mathcal{A}^{\varepsilon}(f), y_{d, 1}^{\varepsilon}=\mathcal{A}^{\varepsilon}\left(y_{d, 1}\right), y_{d, 2}^{\varepsilon}=\mathcal{A}^{\varepsilon}\left(y_{d, 2}\right)$ vanish for all modes $m \in \mathbb{N}$, and for $m=0$ they are given by

$$
\hat{f}_{\varepsilon, 0}=\left\{\begin{array}{lr}
\hat{f} & \text { in } K \\
0 & \text { in }(Z / \varepsilon) \backslash K,
\end{array} \hat{y}_{d, 1,0}^{\varepsilon}=\left\{\begin{array}{lr}
\hat{y}_{d, 1}^{\varepsilon} & \text { in } K \\
0 & \text { in }(Z / \varepsilon) \backslash K,
\end{array} \hat{y}_{d, 2,0}^{\varepsilon}=\left\{\begin{array}{lr}
\hat{y}_{d, 2}^{\varepsilon} & \text { in } K \\
0 & \text { in }(Z / \varepsilon) \backslash K .
\end{array}\right.\right.\right.
$$

Now, the first claim (2.1) follows directly from (1.12) applied to $y_{\varepsilon}$,

$$
-\nabla \cdot\left(A^{\varepsilon}(x) \nabla y_{\varepsilon}(x)\right)+y_{\varepsilon}(x)=\int_{Z / \varepsilon} \sum_{m \in \mathbb{N}_{0}}\left(1+\lambda_{m}^{\varepsilon}(\eta)\right) \hat{y}_{\varepsilon, m}(\eta) \omega_{m}^{\varepsilon}(x ; \eta) \mathrm{d} \eta .
$$

By comparing this expansion with the Bloch expansion of $f^{\varepsilon}+u_{\varepsilon}$ along with (2.4), the claim (2.1) follows. In view of (2.1), the Bloch coefficients of $y_{\varepsilon}$ are given by

$$
\hat{y}_{\varepsilon, m}=\frac{\hat{u}_{\varepsilon, m}(\eta)}{1+\lambda_{m}^{\varepsilon}(\eta)} \quad \forall m \in \mathbb{N} \quad \text { and } \quad \hat{y}_{\varepsilon, 0}=\left\{\begin{array}{lr}
\frac{\hat{f}(\eta)+\hat{u}_{\varepsilon, 0}(\eta)}{1+\lambda_{0}^{\varepsilon}(\eta)} & \text { in } K \\
\frac{\hat{u}_{\varepsilon, 0}(\eta)}{1+\lambda_{0}^{\varepsilon}(\eta)} & \text { in }(Z / \varepsilon) \backslash K .
\end{array}\right.
$$

Making use of (2.6) and (2.4), the claim (2.3) follows directly from Parseval's identity (1.11) applied to $y_{\epsilon}-y_{d, 2}^{\varepsilon}$. Finally, to prove (2.2), we apply (1.12) to $y_{\varepsilon}-y_{d, 1}^{\varepsilon}$ and obtain that

$$
\begin{aligned}
& -\nabla \cdot\left(A^{\varepsilon}(x) \nabla\left(y_{\varepsilon}-y_{d, 1}^{\varepsilon}\right)(x)\right)+\left(y_{\varepsilon}-y_{d, 1}^{\varepsilon}\right)(x) \\
= & \int_{K}\left(1+\lambda_{0}^{\varepsilon}(\eta)\right)\left(\hat{y}_{\varepsilon, 0}-\hat{y}_{d, 1}\right)(\eta) \omega_{0}^{\varepsilon}(x ; \eta) \mathrm{d} \eta+\int_{(Z / \varepsilon) \backslash K}\left(1+\lambda_{0}^{\varepsilon}(\eta)\right) \hat{y}_{\varepsilon, 0}(\eta) \omega_{0}^{\varepsilon}(x ; \eta) \mathrm{d} \eta \\
& +\int_{Z / \varepsilon} \sum_{m \geq 1}\left(1+\lambda_{m}^{\varepsilon}(\eta)\right) \hat{y}_{\varepsilon, m}(\eta) \omega_{m}^{\varepsilon}(x ; \eta) \mathrm{d} \eta .
\end{aligned}
$$

Applying the Plancherel identity for the Bloch expansion to the scalar product $\left(-\nabla \cdot\left(A^{\varepsilon} \nabla\left(y_{\varepsilon}-y_{d, 1}^{\varepsilon}\right)\right)+y_{\varepsilon}-\right.$ $\left.y_{d, 1}^{\varepsilon}, y_{\varepsilon}-y_{d, 1}^{\varepsilon}\right)_{L^{2}\left(\mathbb{R}^{n}\right)}$ and the scalar product of their respective Bloch representations given by (2.7), (2.6), and (2.4), we conclude that $(2.2)$ is valid.

In the next step, we show that for the optimal control $u_{\varepsilon}^{*}$ all Bloch modes with $m \geq 1$ or $\eta \in(Z / \varepsilon) \backslash K$ vanish.

Proposition 2.2. Under the assumptions of Lemma 2.1, it holds that

$$
\begin{aligned}
& u_{\varepsilon}^{*}(x)=\int_{K} \hat{u}_{\varepsilon, 0}^{*}(\eta) \omega_{0}^{\varepsilon}(x ; \eta) \mathrm{d} \eta, \\
& y_{\varepsilon}^{*}(x)=\int_{K} \frac{\hat{f}(\eta)+\hat{u}_{\varepsilon, 0}^{*}(\eta)}{1+\lambda_{0}^{\varepsilon}(\eta)} \omega_{0}^{\varepsilon}(x ; \eta) \mathrm{d} \eta .
\end{aligned}
$$


In particular, $u_{\varepsilon}^{*}=\mathcal{A}^{\varepsilon}\left(\tilde{u}_{\varepsilon}^{*}\right)$ and $y_{\varepsilon}^{*}(x)=\mathcal{A}^{\varepsilon}\left(\tilde{y}_{\varepsilon}^{*}\right)$ with

$$
\begin{aligned}
& \tilde{u}_{\varepsilon}^{*}(x)=\int_{K} \hat{u}_{\varepsilon, 0}^{*}(\eta) e^{2 \pi i \eta \cdot x} \mathrm{~d} \eta, \\
& \tilde{y}_{\varepsilon}^{*}(x)=\int_{K} \frac{\hat{f}(\eta)+\hat{u}_{\varepsilon, 0}^{*}(\eta)}{1+\lambda_{0}^{\varepsilon}(\eta)} e^{2 \pi i \eta \cdot x} \mathrm{~d} \eta .
\end{aligned}
$$

Remark 2.3. As pointed out in Remark 1.12, we do not impose any compact assumption on the admissible control set $U_{a d}^{\varepsilon}$. As a consequence of Proposition 2.2, the optimal control of $\left(\mathrm{P}_{\varepsilon}\right)$ turns out to be the adaption of some function $\tilde{u}_{\varepsilon}^{*}$ with compact support $K$ in Fourier space. This is a strong structural result deduced from Assumptions 1.8 and 1.10. In particular only the lowest Bloch mode with $\eta \in K$ contributes to the solution of $\left(\mathrm{P}_{\varepsilon}\right)$, which reduces the relation between the optimal control $u_{\varepsilon}^{*}$ and the optimal state $y_{\varepsilon}^{*}$ to the classical ansatz of homogenization.

Proof. Our aim is to show that $\hat{u}_{\varepsilon, m}^{*}(\eta)=0$ for all $m \geq 1$ and all $\eta \in Z / \varepsilon$ and that $\hat{u}_{\varepsilon, 0}^{*}(\eta)=0$ for all $\eta \in$ $(Z / \varepsilon) \backslash K$. Indeed, from the representation formula for $J^{\varepsilon}$ (Lem. 2.1), one directly concludes that for $u \in U_{a d}^{\varepsilon}$ the projection $u_{K}$ in the sense of (1.17) satisfies

$$
J^{\varepsilon}\left(u, S_{\epsilon} u\right) \geq J^{\varepsilon}\left(u_{K}, S_{\epsilon} u_{K}\right)
$$

with equality iff $u=u_{K}$. Since by Assumption 1.10 the projection $u_{K}$ of every $u \in U_{a d}^{\varepsilon}$ lies again in $U_{a d}^{\varepsilon}$, the (unique) optimal control satisfies $u_{\varepsilon}^{*}=\left(u_{\varepsilon}^{*}\right)_{K}$, which is the claimed result (2.8). The statement (2.9) is then a direct consequence of (2.8) and (2.1).

Proposition 2.2 states that the optimal control of $\left(\mathrm{P}_{\varepsilon}\right)$ is an adaption $u_{\varepsilon}^{*}=\mathcal{A}^{\varepsilon}\left(\tilde{u}_{\varepsilon}^{*}\right)$, with the Bloch coefficient $\hat{u}_{\varepsilon, 0}^{*}$ supported in $K$. In order to construct a first approximation for $y_{\varepsilon}^{*}$, we exploit the formula (2.9) and the fact that the lowest Bloch eigenvalue $\lambda_{0}$ is analytic in a neighbourhood of $\eta=0$ ( $c f$. Lem. 1.4). In particular, for $M \in \mathbb{N}$ arbitrary, we can expand

$$
\begin{aligned}
\lambda_{0}^{\varepsilon}(\eta)=\frac{1}{\varepsilon^{2}} \lambda_{0}(\varepsilon \eta)= & (2 \pi)^{2} \mathbb{A}_{2}^{*} \cdot \eta^{\otimes 2}+\varepsilon^{2}(2 \pi)^{4} \mathbb{A}_{4}^{*} \cdot \eta^{\otimes 4}+\ldots \\
& +\varepsilon^{2 M-2}(2 \pi)^{2 M} \mathbb{A}_{2 M}^{*} \cdot \eta^{\otimes 2 M}+O\left(\varepsilon^{2 M}|\eta|^{2 M+2}\right),
\end{aligned}
$$

where the error is of order $\varepsilon^{2 M}$ uniformly in $\eta \in K$. With

$$
P_{M}^{\varepsilon}(\eta):=(2 \pi)^{2} \mathbb{A}_{2}^{*} \cdot \eta^{\otimes 2}+\varepsilon^{2}(2 \pi)^{4} \mathbb{A}_{4}^{*} \cdot \eta^{\otimes 4}+\cdots+\varepsilon^{2 M-2}(2 \pi)^{2 M} \mathbb{A}_{2 M}^{*} \cdot \eta^{\otimes 2 M},
$$

we propose the following approximation for $\tilde{y}_{\varepsilon}^{*}$ :

$$
\tilde{y}_{M, a p p}^{*}(x):=\int_{K} \frac{\hat{f}(\eta)+\hat{u}_{\varepsilon, 0}^{*}(\eta)}{1+P_{M}^{\varepsilon}(\eta)} e^{2 \pi i \eta \cdot x} \mathrm{~d} \eta
$$

Indeed, the above function $\tilde{y}_{M, a p p}^{*}$ provides a good approximation for $\tilde{y}_{\varepsilon}^{*}$ in the following sense.

Proposition 2.4. Let $\varepsilon>0$ be such that $1+P_{M}^{\varepsilon}(\eta)>0$ for all $\eta \in K$. Let $\tilde{y}_{\varepsilon}^{*}$ be as in (2.11), and let $\tilde{y}_{M, \text { app }}^{*}$ be defined through (2.14). Then there exists a constant $C>0$ such that

$$
\left\|\tilde{y}_{\varepsilon}^{*}-\tilde{y}_{M, a p p}^{*}\right\|_{L^{2}\left(\mathbb{R}^{n}\right)} \leq C \varepsilon^{2 M}
$$


Proof. Using Parseval's identity for Fourier expansions one finds

$$
\begin{array}{r}
\left\|\tilde{y}_{\varepsilon}^{*}-\tilde{y}_{M, a p p}^{*}\right\|_{L^{2}\left(\mathbb{R}^{n}\right)}^{2}=\int_{K}\left|\hat{f}(\eta)+\hat{u}_{\varepsilon, 0}^{*}(\eta)\right|^{2}\left|\frac{1}{1+\lambda_{0}^{\varepsilon}(\eta)}-\frac{1}{1+P_{M}^{\varepsilon}(\eta)}\right|^{2} \mathrm{~d} \eta \\
\leq\left\|\frac{1}{1+\lambda_{0}^{\varepsilon}(\eta)}-\frac{1}{1+P_{M}^{\varepsilon}(\eta)}\right\|_{L^{\infty}(K)}^{2} \int_{K}\left|\hat{f}(\eta)+\hat{u}_{\varepsilon, 0}^{*}(\eta)\right|^{2} \mathrm{~d} \eta \\
\leq \tilde{C} \varepsilon^{4 M}\left\|\hat{f}+\hat{u}_{\varepsilon, 0}^{*}\right\|_{L^{2}(K)}^{2} \underbrace{\leq}_{(1.11)} 2 \tilde{C} \varepsilon^{4 M}\left(\|f\|_{L^{2}\left(\mathbb{R}^{n}\right)}^{2}+\left\|u_{\varepsilon}^{*}\right\|_{L^{2}\left(\mathbb{R}^{n}\right)}^{2}\right) \underbrace{\leq}_{\text {Lemma } 1.13} C^{2} \varepsilon^{4 M},
\end{array}
$$

where for the second inequality we exploited that the error between $P_{M}^{\varepsilon}$ and $\lambda_{0}^{\varepsilon}$ is of order $\varepsilon^{2 M}$ uniformly in $\eta \in K$.

By a direct calculation, the approximation $\tilde{y}_{M, a p p}^{*}$ is a solution to the following $(2 M)$ th order constant coefficient equation

$$
\sum_{k=1}^{M} \varepsilon^{2 k-2}(-1)^{k} \mathbb{A}_{2 k}^{*} D^{2 k} \tilde{y}_{M, a p p}^{*}(x)+\tilde{y}_{M, a p p}^{*}(x)=f(x)+\tilde{u}_{\varepsilon}^{*}(x),
$$

where $\tilde{u}_{\varepsilon}^{*}$ was defined in (2.10) satisfying $u_{\varepsilon}^{*}=\mathcal{A}^{\varepsilon}\left(\tilde{u}_{\varepsilon}^{*}\right)$. It is therefore reasonable to regard (1.27) as a candidate for an effective PDE-constraint. The effective objective functional (1.25) can be justified as follows. Let $u \in U_{a d}^{*}$ be a function with compact support $K$ in Fourier space, i.e.,

$$
u(x)=\int_{K} \hat{u}(\eta) e^{2 \pi i \eta \cdot x} \mathrm{~d} \eta .
$$

We readily know that the Bloch coefficients $\hat{u}_{\varepsilon, m}$ of the adaption $\mathcal{A}^{\varepsilon}(u) \in U_{a d}^{\varepsilon}$ satisfy $\hat{u}_{\varepsilon, m}=0$ for all $m \geq 1$ and $\hat{u}_{\varepsilon, 0}=\hat{u}$. Therefore, as a consequence of Lemma 2.1, we obtain

$$
\begin{aligned}
J^{\varepsilon}\left(\mathcal{A}^{\varepsilon}(u), S_{\epsilon} \mathcal{A}^{\varepsilon}(u)\right)= & \hat{J}^{\varepsilon}(\hat{u})=\frac{\mu_{1}}{2} \int_{K}\left|\frac{\hat{f}(\eta)+\hat{u}(\eta)}{1+\lambda_{0}^{\varepsilon}(\eta)}-\hat{y}_{d, 1}(\eta)\right|^{2}\left(1+\lambda_{0}^{\varepsilon}(\eta)\right) \mathrm{d} \eta \\
& +\frac{\mu_{2}}{2} \int_{K}\left|\frac{\hat{f}(\eta)+\hat{u}(\eta)}{1+\lambda_{0}^{\varepsilon}(\eta)}-\hat{y}_{d, 2}(\eta)\right|^{2} \mathrm{~d} \eta+\frac{\kappa}{2} \int_{K}|\hat{u}(\eta)|^{2} \mathrm{~d} \eta
\end{aligned}
$$

In order to find a candidate for the effective cost functional, we proceed analogously to the derivation of the effective PDE-constraint by formally replacing all $\left(1+\lambda_{0}^{\varepsilon}(\eta)\right)$-terms in $(2.16)$ by $1+P_{M}^{\varepsilon}(\eta)$. This leads to the following candidate for the effective energy

$$
\begin{aligned}
\hat{J}_{M}^{*}(\hat{u})= & \frac{\mu_{1}}{2} \int_{K}\left|\frac{\hat{f}(\eta)+\hat{u}(\eta)}{1+P_{M}^{\varepsilon}(\eta)}-\hat{y}_{d, 1}(\eta)\right|^{2}\left(1+P_{M}^{\varepsilon}(\eta)\right) \mathrm{d} \eta \\
& +\frac{\mu_{2}}{2} \int_{K}\left|\frac{\hat{f}(\eta)+\hat{u}(\eta)}{1+P_{M}^{\varepsilon}(\eta)}-\hat{y}_{d, 2}(\eta)\right|^{2} \mathrm{~d} \eta+\frac{\kappa}{2} \int_{K}|\hat{u}(\eta)|^{2} \mathrm{~d} \eta .
\end{aligned}
$$

For all sufficiently small $\varepsilon>0$ and $u \in U_{a d}^{*}$ (see (1.28) for its definition), Proposition 2.6 below yields the wellposedness of the effective state equation (1.27) and the explicit formula (2.22) for the corresponding solution. 
Applying (2.22), a direct calculation in Fourier space for (1.25) implies for all sufficiently small $\varepsilon>0$ that

$$
J_{M}^{*}(u, y)=\hat{J}_{M}^{*}(\hat{u})
$$

holds for every $u \in U_{a d}^{*}$ with the associated effective state $y$ of (1.27). In this way, we obtain the effective model $\left(\mathrm{P}_{M}^{*}\right)$.

\subsection{Well-posedness of $\left(\mathrm{P}_{M}^{*}\right)$}

The goal of this section is to prove the existence of a unique optimal solution to $\left(\mathrm{P}_{M}^{*}\right)$, provided that $\varepsilon>0$ is sufficiently small. The main difficulty lies in the well-posedness of the effective equation

$$
\mathcal{L}_{M}^{*}(\tilde{y}):=\sum_{k=1}^{M} \varepsilon^{2 k-2}(-1)^{k} \mathbb{A}_{2 k}^{*} D^{2 k} \tilde{y}(x)+\tilde{y}(x)=f(x)+u(x),
$$

where the highest-order operator $\mathbb{A}_{2 M}^{*} D^{2 M}$ might have no or even the wrong sign. Indeed, while $\mathbb{A}_{2}^{*}$ is positive definite, for $\mathbb{A}_{4}^{*}$ it has been shown in [15] that this tensor is negative semidefinite. In particular, for $M=2$, (1.27) reads as

$$
-\mathbb{A}_{2}^{*} D^{2} \tilde{y}(x)+\varepsilon^{2} \mathbb{A}_{4}^{*} D^{4} \tilde{y}(x)+\tilde{y}(x)=f(x)+u(x) .
$$

This fourth-order equation is ill-posed for general right hand sides. However, in our setting, $f$ and every control $\tilde{u} \in U_{a d}^{*}$ have compact support $K$ in Fourier space. Whenever $\varepsilon>0$ is sufficiently small the effective equation can be uniquely solved. The well-posedness is based on the following algebraic observation and the fact that the first effective tensor $\mathbb{A}_{2}^{*}$ is positive definite, i.e., $\mathbb{A}_{2}^{*} \cdot \eta^{\otimes 2} \geq \lambda|\eta|^{2}$ for some $\lambda>0$.

Lemma 2.5. Let $K \in \mathbb{R}^{n}$ be a given compact set and let $M \in \mathbb{N}$ be fixed. Let $\lambda>0$ be the ellipticity constant of $\mathbb{A}_{2}^{*}$ as above. Then there exists $\varepsilon_{M}>0$ such that for every $0<\varepsilon<\varepsilon_{M}$ and every $N \in \mathbb{N}$ with $N \leq M$

$$
P_{N}^{\varepsilon}(\eta) \geq \frac{\lambda}{2}|\eta|^{2} \quad \text { for all } \eta \in K
$$

where $P_{N}^{\varepsilon}$ is the polynomial defined in (2.13).

Proof. Since $\mathbb{A}_{2}^{*} \cdot \eta^{\otimes 2} \geq \lambda|\eta|^{2}$, it is sufficient to prove that there exists some $\varepsilon_{M}>0$ such that for every $0<\varepsilon<$ $\varepsilon_{M}$ and every $N \leq M$

$$
\varepsilon^{2}(2 \pi)^{4} \mathbb{A}_{4}^{*} \cdot \eta^{\otimes 4}+\cdots+\varepsilon^{2 N-2}(2 \pi)^{2 N} \mathbb{A}_{2 N}^{*} \cdot \eta^{\otimes 2 N} \geq-\frac{\lambda}{2}|\eta|^{2}
$$

Indeed,

$$
\sum_{k=2}^{N}\left|\varepsilon^{2 k-2}(2 \pi)^{2 k} \mathbb{A}_{2 k}^{*} \cdot \eta^{\otimes 2 k}\right| \leq(2 \pi)^{2 N}|\eta|^{2}\left(\sum_{k=2}^{N}(\varepsilon|\eta|)^{2 k-2}\left\|\mathbb{A}_{2 k}^{*}\right\|\right)
$$

where $\left\|\mathbb{A}_{2 k}^{*}\right\|$ denotes an appropriate norm of the tensor $\mathbb{A}_{2 k}^{*}$. Since $\eta \in K$ varies in a bounded set, we can choose $\varepsilon_{M}>0$ such that for every $0<\varepsilon<\varepsilon_{M}$

$$
(2 \pi)^{2 N} \sum_{k=2}^{N}(\varepsilon|\eta|)^{2 k-2}\left\|\mathbb{A}_{2 k}^{*}\right\| \leq(2 \pi)^{2 M} \sum_{k=2}^{M}(\varepsilon|\eta|)^{2 k-2}\left\|\mathbb{A}_{2 k}^{*}\right\|<\frac{\lambda}{2} .
$$


Then $\varepsilon_{M}$ satisfies the requirements of the lemma.

We emphasize that the threshold $\varepsilon_{M}$ depends on the compact set $K$, the order of approximation $M$ and the effective coefficients $\mathbb{A}_{2}^{*}, \ldots, \mathbb{A}_{2 M}^{*}$. With the above auxiliary lemma at hand we can now conclude the wellposedness of the effective equation (1.27).

Proposition 2.6 (Well-posedness of (1.27)). Suppose that Assumption 1.8 holds. Let $M \in \mathbb{N}$ and let $\varepsilon_{M}$ be as in Lemma 2.5. Then, for every $0<\varepsilon<\varepsilon_{M}$ and every $u \in L^{2}\left(\mathbb{R}^{n}\right)$ with Fourier transform $\hat{u}$ satisfying $\operatorname{supp}(\hat{u}) \subset K$, it holds that:

i) There exists a unique weak solution $y \in H^{k}\left(\mathbb{R}^{n}\right)$ for all $k \in \mathbb{N}$ to the effective state equation (1.27).

ii) The solution $y$ satisfies the a priori estimate

$$
\|y\|_{L^{2}\left(\mathbb{R}^{n}\right)}^{2}+\lambda\|\nabla y\|_{L^{2}\left(\mathbb{R}^{n}\right)}^{2} \leq\|f+u\|_{L^{2}\left(\mathbb{R}^{n}\right)}^{2} .
$$

Remark 2.7. By the definition of the effective admissible control set $U_{a d}^{*}$ in (1.28), every $u \in U_{a d}^{*}$ automatically satisfies the requirement of Proposition 2.6.

Proof of Proposition 2.6. To show existence, let us consider

$$
y(x):=\int_{K} \frac{\hat{f}(\eta)+\hat{u}(\eta)}{1+P_{M}^{\varepsilon}(\eta)} e^{2 \pi i \eta \cdot x} \mathrm{~d} \eta .
$$

As a function with compact support in Fourier space, $y$ belongs to $H^{k}\left(\mathbb{R}^{n}\right)$ for all $k \in \mathbb{N}$. By a direct calculation in Fourier space one finds that (2.22) is a solution to the effective equation (1.27). It remains to prove the a priori estimate (2.21), from which uniqueness directly follows. Therefore note that the Fourier transform $\hat{y}$ of every solution satisfies

$$
\left(1+P_{M}^{\varepsilon}\right) \hat{y}=\hat{f}+\hat{u}
$$

Testing the above relation with $\hat{y}$, taking into account $\hat{f}(\eta)=\hat{u}(\eta)=\hat{y}(\eta)=0$ for $\eta \notin K$, and exploiting Lemma 2.5 provides that

$$
\begin{aligned}
\int_{\mathbb{R}^{n}}\left(1+\frac{\lambda}{2}|\eta|^{2}\right)|\hat{y}(\eta)|^{2} \mathrm{~d} \eta & \leq \int_{K}\left(1+P_{M}^{\varepsilon}(\eta)\right)|\hat{y}(\eta)|^{2} \mathrm{~d} \eta=\int_{K}(\hat{f}(\eta)+\hat{u}(\eta)) \overline{\hat{y}(\eta)} \mathrm{d} \eta \\
& \leq\|\hat{f}+\hat{u}\|_{L^{2}(K)}\|\hat{y}\|_{L^{2}(K)} \leq \frac{1}{2}\left(\|\hat{f}+\hat{u}\|_{L^{2}(K)}^{2}+\|\hat{y}\|_{L^{2}\left(\mathbb{R}^{n}\right)}^{2}\right),
\end{aligned}
$$

where $\overline{\hat{y}}$ denotes the complex conjugate of $\hat{y}$. By Parseval's identity one immediately concludes the desired estimate (2.21).

We have found that the PDE-constraint (1.27) is well-posed. To prove that the corresponding effective optimal control problem $\left(\mathrm{P}_{M}^{*}\right)$ has a unique optimal solution, we reformulate $\left(\mathrm{P}_{M}^{*}\right)$ in terms of the Fourier transform. In the same manner the original problem $\left(\mathrm{P}_{\varepsilon}\right)$ can be rewritten in terms of the Bloch transform, which is particularly important for the error analysis in Section 3. 
Proposition 2.8 (Reformulation of the optimal control problems). Let Assumptions 1.8 and 1.10 hold, and let $U_{a d}^{*}$ be the effective admissible set from (1.28). Then

$$
\begin{aligned}
\hat{U}_{a d} & :=\left\{\hat{u} \in L^{2}(K ; \mathbb{C}) \mid u_{F} \in U_{a d}^{*} \text { with } u_{F}(x)=\int_{K} \hat{u}(\eta) e^{2 \pi i \eta \cdot x} \mathrm{~d} \eta\right\} \\
& =\left\{\hat{u} \in L^{2}(K ; \mathbb{C}) \mid u_{B} \in\left(U_{a d}^{\varepsilon}\right)_{K} \text { with } u_{B}(x)=\int_{K} \hat{u}(\eta) \omega_{0}^{\varepsilon}(x ; \eta) \mathrm{d} \eta\right\} .
\end{aligned}
$$

\section{Furthermore:}

(i) The optimal control problem $\left(\mathrm{P}_{\varepsilon}\right)$ is equivalent to

$$
\begin{array}{r}
\min _{\hat{u} \in \hat{U}_{a d}} \hat{J}^{\varepsilon}(\hat{u}):=\frac{\mu_{1}}{2} \int_{K}\left|\frac{\hat{f}(\eta)+\hat{u}(\eta)}{1+\lambda_{0}^{\varepsilon}(\eta)}-\hat{y}_{d, 1}(\eta)\right|^{2}\left(1+\lambda_{0}^{\varepsilon}(\eta)\right) \mathrm{d} \eta \\
+\frac{\mu_{2}}{2} \int_{K}\left|\frac{\hat{f}(\eta)+\hat{u}(\eta)}{1+\lambda_{0}^{\varepsilon}(\eta)}-\hat{y}_{d, 2}(\eta)\right|^{2} \mathrm{~d} \eta+\frac{\kappa}{2} \int_{K}|\hat{u}(\eta)|^{2} \mathrm{~d} \eta
\end{array}
$$

in the sense that $\left(u_{\varepsilon}^{*}, y_{\varepsilon}^{*}\right)$ solves $\left(\mathrm{P}_{\varepsilon}\right)$ if and only if the Bloch transform of $u_{\varepsilon}^{*}$ solves $(2.24)$

(ii) If $0<\varepsilon<\varepsilon_{M}$ with $\varepsilon_{M}$ as in Lemma 2.5, then the effective optimal control problem $\left(\mathrm{P}_{M}^{*}\right)$ is equivalent to

$$
\begin{array}{r}
\min _{\hat{u} \in \hat{U}_{a d}} \hat{J}_{M}^{*}(\hat{u}):=\frac{\mu_{1}}{2} \int_{K}\left|\frac{\hat{f}(\eta)+\hat{u}(\eta)}{1+P_{M}^{\varepsilon}(\eta)}-\hat{y}_{d, 1}(\eta)\right|^{2}\left(1+P_{M}^{\varepsilon}(\eta)\right) \mathrm{d} \eta \\
+\frac{\mu_{2}}{2} \int_{K}\left|\frac{\hat{f}(\eta)+\hat{u}(\eta)}{1+P_{M}^{\varepsilon}(\eta)}-\hat{y}_{d, 2}(\eta)\right|^{2} \mathrm{~d} \eta+\frac{\kappa}{2} \int_{K}|\hat{u}(\eta)|^{2} \mathrm{~d} \eta
\end{array}
$$

in the sense that $\left(u_{M}^{*}, y_{M}^{*}\right)$ solves $\left(\mathrm{P}_{M}^{*}\right)$ if and only if the Fourier transform of $u_{M}^{*}$ solves (2.25).

We emphasize that the set $\hat{U}_{a d}$ in (2.23) may depend on $\epsilon$.

Proof. By virtue of (1.28), (1.17), and $\left(U_{a d}^{\varepsilon}\right)_{K} \subset U_{a d}^{\varepsilon}$, we have $\mathcal{A}^{\varepsilon}\left(U_{a d}^{*}\right)=\left(U_{a d}^{\varepsilon}\right)_{K}$. Furthermore, since for functions with compact support in Fourier space the adaption $\mathcal{A}^{\varepsilon}$ replaces the Fourier basis function $e^{2 \pi i \eta \cdot x}$ in the expansion by the Bloch function $\omega_{0}^{\varepsilon}(x ; \eta)$, it follows that $u_{B}=\mathcal{A}^{\varepsilon}\left(u_{F}\right)$. In particular the two sets in $(2.23)$ are equal.

To prove (i), we note that by Proposition 2.2 the optimal control $u_{\varepsilon}^{*}$ of $\left(\mathrm{P}_{\varepsilon}\right)$ lies in the projection $\left(U_{a d}^{\varepsilon}\right)_{K}$. This fact along with $\mathcal{A}^{\varepsilon}\left(U_{a d}^{*}\right)=\left(U_{a d}^{\varepsilon}\right)_{K}$ leads to

$$
\begin{array}{r}
u_{\varepsilon}^{*} \text { solves } \min _{u \in U_{a d}^{\varepsilon}} J^{\varepsilon}\left(u, S_{\epsilon} u\right) \Leftrightarrow u_{\varepsilon}^{*} \text { solves } \min _{u \in\left(U_{a d}^{\varepsilon}\right)_{K}} J^{\varepsilon}\left(u, S_{\epsilon} u\right) \\
\Leftrightarrow \quad u_{\varepsilon}^{*}=\mathcal{A}^{\varepsilon}\left(u_{F}^{*}\right) \text { and } u_{F}^{*} \text { solves } \min _{u_{F} \in U_{a d}^{*}} J^{\varepsilon}\left(\mathcal{A}^{\varepsilon}\left(u_{F}\right), S_{\epsilon} \mathcal{A}^{\varepsilon}\left(u_{F}\right)\right) \\
\underbrace{\Leftrightarrow}_{(1.28),(2.16),(2.23)} u_{\varepsilon}^{*}=\int_{K} \hat{u}^{*}(\eta) \omega_{0}^{\varepsilon}(\cdot ; \eta) \mathrm{d} \eta \text { and } \hat{u}^{*} \text { solves } \min _{\hat{u} \in \hat{U}_{a d}} \hat{J}^{\varepsilon}(\hat{u}) .
\end{array}
$$

The second claim (ii) follows immediately from (2.18) and the definition (2.23).

Remark 2.9. Due to its quadratic form, the effective energy $\hat{J}_{M}^{*}: L^{2}(K ; \mathbb{C}) \rightarrow \mathbb{R}$ is convex and continuous. 
Finally we prove that the admissible set $\hat{U}_{a d}$ defined in (2.23) is nonempty, convex, and closed. Together with Remark 2.9 this yields the well-posedness of the effective optimal control problem $\left(\mathrm{P}_{M}^{*}\right)$ provided $\varepsilon$ is sufficiently small, i.e., $\varepsilon<\varepsilon_{M}$ and $K \subset Z / \varepsilon$.

Proposition 2.10. Let $U_{a d}^{\varepsilon}$ satisfy Assumption 1.10. Then the set $\hat{U}_{a d}$ defined as in $(2.23)$ is nonempty, convex, and closed in $L^{2}(K ; \mathbb{C})$.

Proof. The set $\hat{U}_{a d}$ is nonempty since for every $u \in U_{a d}^{\varepsilon} \neq \emptyset$ the projection $u_{K}$ satisfies $u_{K} \in\left(U_{a d}^{\varepsilon}\right)_{K}$ with the Bloch coefficient belonging to $\hat{U}_{a d}$ according to $(2.23)$.

In view of $(2.23)$, the convexity of $\hat{U}_{a d} \subset L^{2}(K ; \mathbb{C})$ holds if $U_{a d}^{*} \subset L^{2}\left(\mathbb{R}^{n}\right)$ is convex. By the linearity of the adaption $\mathcal{A}^{\varepsilon}$ and the definition of $U_{a d}^{*}(1.28)$, the convexity of $U_{a d}^{\varepsilon} \subset L^{2}\left(\mathbb{R}^{n}\right)$ implies immediately the convexity of $U_{a d}^{*} \subset L^{2}\left(\mathbb{R}^{n}\right)$.

Due to Parseval's identity (1.11), strong convergence of functions in $L^{2}\left(\mathbb{R}^{n}\right)$ is equivalent to strong $L^{2}$ convergence of the corresponding Bloch transforms. Thus, it follows from (1.17), $\left(U_{a d}^{\varepsilon}\right)_{K} \subset U_{a d}^{\varepsilon}$, and the closedness of $U_{a d}^{\varepsilon} \subset L^{2}\left(\mathbb{R}^{n}\right)$ that the projected set $\left(U_{a d}^{\varepsilon}\right)_{K}$ is closed in $L^{2}\left(\mathbb{R}^{n}\right)$, and so by (2.23) the closedness of $\hat{U}_{a d} \subset L^{2}(K ; \mathbb{C})$ follows.

Corollary 2.11. Let Assumptions 1.8 and 1.10 hold. Furthermore, let $M \in \mathbb{N}$ and let $\varepsilon_{M}$ be as in Lemma 2.5. Then, for every $0<\varepsilon<\varepsilon_{M}$, the effective optimal control problem $\left(\mathrm{P}_{M}^{*}\right)$ admits a unique optimal solution $\left(u_{M}^{*}, y_{M}^{*}\right)$.

To conclude this section we specify the effective admissible sets $\hat{U}_{a d}$ and $U_{a d}^{*}$ corresponding to the sets from Example 1.14. At this point, we recall that $u \in L^{2}\left(\mathbb{R}^{d} ; \mathbb{C}\right)$ is real valued if and only if $\hat{u}(-\eta)=\overline{\hat{u}(\eta)}$. An analogous statement holds for the Bloch transforms.

Example 2.12 (Effective admissible sets). Suppose that Assumption 1.8 holds. Let $\hat{U}_{a d}$ be defined through (2.23) and $U_{a d}^{*}$ through (1.28).

(i) For $U_{a d}^{\varepsilon}=L^{2}\left(\mathbb{R}^{n}\right)$ one has

$$
\begin{aligned}
& \hat{U}_{a d}=\left\{L^{2}(K ; \mathbb{C}): \hat{u}(-\eta)=\overline{\hat{u}(\eta)}\right\}, \\
& U_{a d}^{*}=\left\{u \in L^{2}\left(\mathbb{R}^{n}\right) \mid \operatorname{supp}(\hat{u}) \subset K\right\} .
\end{aligned}
$$

(ii) For $U_{a d}^{\varepsilon}:=\left\{u \in L^{2}\left(\mathbb{R}^{n}\right) \mid\|u\|_{L^{2}\left(\mathbb{R}^{n}\right)} \leq L\right\}$ one has due to Pareval's identity

$$
\begin{aligned}
& \hat{U}_{a d}=\left\{\hat{u} \in L^{2}(K ; \mathbb{C}) \mid \hat{u}(-\eta)=\overline{\hat{u}(\eta)},\|\hat{u}\|_{L^{2}(K ; \mathbb{C})} \leq L\right\}, \\
& U_{a d}^{*}=\left\{u \in L^{2}\left(\mathbb{R}^{n}\right) \mid \operatorname{supp}(\hat{u}) \subset K \text { and }\|u\|_{L^{2}\left(\mathbb{R}^{n}\right)} \leq L\right\} .
\end{aligned}
$$

(v) For $U_{a d}^{\varepsilon}=\mathcal{A}^{\varepsilon}\left(U_{a d}\right)$ one has

$$
\begin{aligned}
& \hat{U}_{a d}=\left\{\hat{u} \in L^{2}(K ; \mathbb{C}) \mid \hat{u} \text { is the Fourier transform of some } u \in U_{a d}\right\}, \\
& U_{a d}^{*}=U_{a d} .
\end{aligned}
$$

The specification of the admissible sets for $U_{a d}^{\varepsilon}$ from Example 1.14 in (iii) and (iv) is more subtle and does not allow for a simple form at first sight.

(iii) For $U_{a d}^{\varepsilon}:=\left\{u \in L^{2}\left(\mathbb{R}^{n}\right) \mid\left\|S_{\epsilon} u\right\|_{L^{2}\left(\mathbb{R}^{n}\right)} \leq L\right\}$ one obtains, using Lemma 2.1,

$$
\hat{U}_{a d}=\left\{\hat{u} \in L^{2}(K ; \mathbb{C}) \mid \hat{u}(-\eta)=\overline{\hat{u}(\eta)},\left\|\frac{\hat{f}+\hat{u}}{1+\lambda_{0}^{\varepsilon}}\right\|_{L^{2}(K ; \mathbb{C})} \leq L\right\}
$$




$$
U_{a d}^{*}=\left\{u(x)=\int_{K} \hat{u}(\eta) e^{2 \pi i \eta \cdot x} \mathrm{~d} \eta \mid \hat{u} \in \hat{U}_{a d}\right\}
$$

(iv) For $U_{a d}^{\varepsilon}:=\left\{u \in L^{2}\left(\mathbb{R}^{n}\right) \mid E^{\varepsilon}\left(S_{\epsilon} u\right) \leq L\right\}$ one obtains, using again Lemma 2.1,

$$
\begin{aligned}
\hat{U}_{a d} & =\left\{\hat{u} \in L^{2}(K ; \mathbb{C}) \mid \hat{u}(-\eta)=\overline{\hat{u}(\eta)}, \int_{K} \frac{|\hat{f}+\hat{u}|^{2}(\eta)}{1+\lambda_{0}^{\varepsilon}(\eta)} \mathrm{d} \eta \leq L\right\}, \\
U_{a d}^{*} & =\left\{u(x)=\int_{K} \hat{u}(\eta) e^{2 \pi i \eta \cdot x} \mathrm{~d} \eta \mid \hat{u} \in \hat{U}_{a d}\right\} .
\end{aligned}
$$

The characterization of the effective set $\hat{U}_{a d}$ for the above two examples uses the $\varepsilon$-dependent Bloch eigenvalue $\lambda_{0}^{\varepsilon}$. To derive a more practicable condition one can again replace $\lambda_{0}^{\varepsilon}$ by its $M$ th order Taylor expansion $P_{M}^{\varepsilon}$. The resulting approximative admissible set $\hat{U}_{a d}^{M}$ is close to $\hat{U}_{a d}$. Due to stability of the optimal control problem the minimization can be performed on this approximative set without loosing the approximation quality. Recalling that solutions to the effective PDE-constaint (1.27) have the Fourier transform $\hat{y}=\frac{\hat{f}+\hat{u}}{1+P_{M}^{\varepsilon}}$, it follows that

$$
\begin{aligned}
\hat{U}_{a d}^{M} & :=\left\{\hat{u} \in L^{2}(K ; \mathbb{C}) \mid \hat{u}(-\eta)=\overline{\hat{u}(\eta)},\left\|\frac{\hat{f}+\hat{u}}{1+P_{M}^{\varepsilon}}\right\|_{L^{2}(K ; \mathbb{C})} \leq L\right\} \\
& =\left\{\hat{u} \in L^{2}(K, \mathbb{C}) \mid \hat{u}(-\eta)=\overline{\hat{u}(\eta)},\|\hat{y}\|_{L^{2}(K ; \mathbb{C})} \leq L\right\} .
\end{aligned}
$$

(iv) In view of the definition $(1.26)$ of $E_{M}^{*}$, denoting by $\mathcal{F}$ the Fourier transform, one obtains

$$
\begin{aligned}
\hat{U}_{a d}^{M} & :=\left\{\hat{u} \in L^{2}(K ; \mathbb{C}) \mid \hat{u}(-\eta)=\overline{\hat{u}(\eta)}, \int_{K} \frac{|\hat{f}+\hat{u}|^{2}(\eta)}{1+P_{M}^{\varepsilon}(\eta)} \mathrm{d} \eta \leq L\right\} \\
& =\left\{\left.\hat{u} \in L^{2}(K ; \mathbb{C})\left|\hat{u}(-\eta)=\overline{\hat{u}(\eta)}, \int_{K}\right| \hat{y}\right|^{2}(\eta)\left(1+P_{M}^{\varepsilon}(\eta)\right) \mathrm{d} \eta \leq L\right\} \\
& =\left\{\hat{u} \in L^{2}(K ; \mathbb{C}) \mid \hat{u}(-\eta)=\overline{\hat{u}(\eta)}, \hat{u}=\mathcal{F}(u) \text { and } E_{M}^{*}(y) \leq L, \text { where } y \text { solves }(1.27)\right\} .
\end{aligned}
$$

\section{ERror ESTIMATES}

Proposition 2.8 allows to rewrite the original and the effective optimal control problems in terms of the Fourier/Bloch transforms. The fact that both minimization problems (2.24) and (2.25) are constrained by the same admissible set $\hat{U}_{a d}$ enables us to compare them by means of the corresponding variational inequalities.

In all what follows, we assume that the order of approximation $M \in \mathbb{N}$ is fixed and that $\varepsilon>0$ is sufficiently small, i.e. $\varepsilon<\varepsilon_{M}$ and such that $K \subset Z / \varepsilon$. We will see that the unique solution $\hat{u}_{M}^{*} \in \hat{U}_{a d}$ of (2.25) is a good approximation the Bloch transform $\hat{u}_{\varepsilon, 0}^{*}$ of the optimal control $u_{\varepsilon}^{*}$ of $\left(\mathrm{P}_{\varepsilon}\right)$, which is exactly the unique solution

to (2.24). This relies on the fact that the cost functionals $\hat{J}^{\varepsilon}$ and $\hat{J}_{M}^{*}$ in (2.24) and (2.25) differ only by the factor $1+\lambda_{0}^{\varepsilon}$, which in $\hat{J}_{M}^{*}$ is replaced by $1+P_{M}^{\varepsilon}$. As a consequence, the (unique) optimal control of $\left(\mathrm{P}_{M}^{*}\right)$, which is according to Proposition 2.8 given by

$$
u_{M}^{*}(x)=\int_{K} \hat{u}_{M}^{*}(\eta) e^{2 \pi i \eta \cdot x} \mathrm{~d} \eta
$$


and the corresponding optimal state $y_{M}^{*}$, given by the solution to the effective equation

$$
\sum_{k=1}^{M} \varepsilon^{2 k-2}(-1)^{k} \mathbb{A}_{2 k}^{*} D^{2 k} y_{M}^{*}(x)+y_{M}^{*}(x)=f(x)+u_{M}^{*}(x),
$$

satisfy the desired approximation (1.29) with $\left(\tilde{u}_{\epsilon}^{*}, \tilde{y}_{\epsilon}^{*}\right)$ as in Proposition 2.2. Let us now prove our first main result, which states the approximation property of $\hat{u}_{M}^{*}$ :

Theorem 3.1 (Approximation of the optimal control). Let $\hat{u}_{\varepsilon, 0}^{*} \in \hat{U}_{a d}$ and $\hat{u}_{M}^{*} \in \hat{U}_{a d}$ denote, respectively, the unique solutions to (2.24) and (2.25). Then

$$
\left\|\hat{u}_{\varepsilon, 0}^{*}-\hat{u}_{M}^{*}\right\|_{L^{2}(K ; \mathbb{C})} \leq C \varepsilon^{2 M}
$$

for some $\varepsilon$-independent constant $C>0$.

We emphasize that the constant $C$ in Theorem 3.1 may depend on the order of approximation $M$ and the compact set $K$.

Proof of Theorem 3.1. As the optimization problems (2.24) and (2.25) are convex with directionally differentiable cost funcitonals, their solutions $\hat{u}_{\varepsilon, 0}^{*}$ and $\hat{u}_{M}^{*}$ satisfy the following variational inequalities

$$
\begin{aligned}
& \int_{K} \operatorname{Re}\left[\left(D \hat{J}^{\varepsilon}\left(\hat{u}_{\varepsilon, 0}^{*}\right)\right)(\eta)\left(\overline{\hat{u}}-\overline{\hat{u}_{\varepsilon, 0}^{*}}\right)(\eta)\right] \mathrm{d} \eta \geq 0 \quad \text { for all } \hat{u} \in \hat{U}_{a d}, \\
& \int_{K} \operatorname{Re}\left[\left(D \hat{J}_{M}^{*}\left(\hat{u}_{M}^{*}\right)\right)(\eta)\left(\overline{\hat{u}}-\overline{\hat{u}_{M}^{*}}\right)(\eta)\right] \mathrm{d} \eta \geq 0 \quad \text { for all } \hat{u} \in \hat{U}_{a d}
\end{aligned}
$$

where Re denotes the real part and

$$
\begin{gathered}
D \hat{J}^{\varepsilon}(\hat{u}):=\mu_{1}\left(\frac{\hat{f}+\hat{u}}{1+\lambda_{0}^{\varepsilon}}-\hat{y}_{d, 1}\right)+\mu_{2}\left(\frac{\hat{f}+\hat{u}}{1+\lambda_{0}^{\varepsilon}}-\hat{y}_{d, 2}\right)\left(1+\lambda_{0}^{\varepsilon}\right)^{-1}+\kappa \hat{u}, \\
D \hat{J}_{M}^{*}(\hat{u}):=\mu_{1}\left(\frac{\hat{f}+\hat{u}}{1+P_{M}^{\varepsilon}}-\hat{y}_{d, 1}\right)+\mu_{2}\left(\frac{\hat{f}+\hat{u}}{1+P_{M}^{\varepsilon}}-\hat{y}_{d, 2}\right)\left(1+P_{M}^{\varepsilon}\right)^{-1}+\kappa \hat{u} .
\end{gathered}
$$

Taking $\hat{u}_{\varepsilon, 0}^{*}$ as a test function in (3.5) and $\hat{u}_{M}^{*}$ as test function in (3.4), we obtain

$$
\begin{gathered}
\int_{K} \operatorname{Re}\left[\left(\mu_{1}\left(\frac{\hat{f}+\hat{u}_{M}^{*}}{1+P_{M}^{\varepsilon}}-\hat{y}_{d, 1}\right)+\mu_{2}\left(\frac{\hat{f}+\hat{u}_{M}^{*}}{1+P_{M}^{\varepsilon}}-\hat{y}_{d, 2}\right)\left(1+P_{M}^{\varepsilon}\right)^{-1}+\kappa \hat{u}_{M}^{*}\right)\left(\overline{\hat{u}_{\varepsilon, 0}^{*}}-\overline{\hat{u}_{M}^{*}}\right)\right](\eta) \mathrm{d} \eta \geq 0, \\
\int_{K} \operatorname{Re}\left[\left(\mu_{1}\left(\frac{\hat{f}+\hat{u}_{\varepsilon, 0}^{*}}{1+\lambda_{0}^{\varepsilon}}-\hat{y}_{d, 1}\right)+\mu_{2}\left(\frac{\hat{f}+\hat{u}_{\varepsilon, 0}^{*}}{1+\lambda_{0}^{\varepsilon}}-\hat{y}_{d, 2}\right)\left(1+\lambda_{0}^{\varepsilon}\right)^{-1}+\kappa \hat{u}_{\varepsilon, 0}^{*}\right)\left(\overline{\hat{u}_{M}^{*}}-\overline{\hat{u}_{\varepsilon, 0}^{*}}\right)\right](\eta) \mathrm{d} \eta \geq 0 .
\end{gathered}
$$

Summing up the two inequalities provides

$$
\begin{aligned}
& \kappa \int_{K}\left|\hat{u}_{\varepsilon, 0}^{*}(\eta)-\hat{u}_{M}^{*}(\eta)\right|^{2} \mathrm{~d} \eta \\
\leq & \mu_{1} \int_{K} \operatorname{Re}\left[\left(\frac{\hat{f}+\hat{u}_{M}^{*}}{1+P_{M}^{\varepsilon}}-\frac{\hat{f}+\hat{u}_{\varepsilon, 0}^{*}}{1+\lambda_{0}^{\varepsilon}}\right)\left(\overline{\hat{u}_{\varepsilon, 0}^{*}}-\overline{\hat{u}_{M}^{*}}\right)\right](\eta) \mathrm{d} \eta \\
& +\mu_{2} \int_{K} \operatorname{Re}\left[\left(\frac{\hat{f}+\hat{u}_{M}^{*}-\left(1+P_{M}^{\varepsilon}\right) \hat{y}_{d, 2}}{\left(1+P_{M}^{\varepsilon}\right)^{2}}-\frac{\hat{f}+\hat{u}_{\varepsilon, 0}^{*}-\left(1+\lambda_{0}^{\varepsilon}\right) \hat{y}_{d, 2}}{\left(1+\lambda_{0}^{\varepsilon}\right)^{2}}\right)\left(\overline{\hat{u}_{\varepsilon, 0}^{*}}-\overline{\hat{u}_{M}^{*}}\right)\right](\eta) \mathrm{d} \eta .
\end{aligned}
$$


Concerning the first term on the right hand side of (3.6) we note that

$$
\frac{\hat{f}+\hat{u}_{M}^{*}}{1+P_{M}^{\varepsilon}}-\frac{\hat{f}+\hat{u}_{\varepsilon, 0}^{*}}{1+\lambda_{0}^{\varepsilon}}=\left(\hat{f}+\hat{u}_{\varepsilon, 0}^{*}\right)\left(\frac{1}{1+P_{M}^{\varepsilon}}-\frac{1}{1+\lambda_{0}^{\varepsilon}}\right)+\frac{1}{1+P_{M}^{\varepsilon}}\left(\hat{u}_{M}^{*}-\hat{u}_{\varepsilon, 0}^{*}\right)
$$

and thus, exploiting that

$$
\frac{1}{1+P_{M}^{\varepsilon}}\left(\hat{u}_{M}^{*}-\hat{u}_{\varepsilon, 0}^{*}\right)\left(\overline{\hat{u}_{\varepsilon, 0}^{*}}-\overline{\hat{u}_{M}^{*}}\right)=-\frac{1}{1+P_{M}^{\varepsilon}}\left|\hat{u}_{M}^{*}-\hat{u}_{\varepsilon, 0}^{*}\right|^{2} \leq 0
$$

it follows that

$$
\begin{aligned}
& \mu_{1} \int_{K} \operatorname{Re}\left[\left(\frac{\hat{f}+\hat{u}_{M}^{*}}{1+P_{M}^{\varepsilon}}-\frac{\hat{f}+\hat{u}_{\varepsilon, 0}^{*}}{1+\lambda_{0}^{\varepsilon}}\right)\left({\overline{u_{\varepsilon, 0}^{*}}}^{*}-\overline{\hat{u}_{M}^{*}}\right)\right](\eta) \mathrm{d} \eta \\
& \leq \mu_{1}\left(\sup _{\eta \in K}\left|\frac{1}{1+P_{M}^{\varepsilon}(\eta)}-\frac{1}{1+\lambda_{0}^{\varepsilon}(\eta)}\right|\right)\left\|\hat{u}_{\varepsilon, 0}^{*}-\hat{u}_{M}^{*}\right\|_{L^{2}(K ; \mathbb{C})}\left\|\hat{f}+\hat{u}_{\varepsilon, 0}^{*}\right\|_{L^{2}(K ; \mathbb{C})} \\
& \leq C \varepsilon^{2 M}\left\|\hat{u}_{\varepsilon, 0}^{*}-\hat{u}_{M}^{*}\right\|_{L^{2}(K ; \mathbb{C})}\left\|\hat{f}+\hat{u}_{\varepsilon, 0}^{*}\right\|_{L^{2}(K ; \mathbb{C})},
\end{aligned}
$$

where in the last step we have used that $\left|P_{M}^{\varepsilon}(\eta)-\lambda_{0}^{\varepsilon}(\eta)\right| \leq C \varepsilon^{2 M}$ uniformly in $\eta \in K$. For the the second term on the right hand side of (3.6) we argue analogously,

$$
\begin{aligned}
& \mu_{2} \int_{K} \operatorname{Re}\left[\left(\frac{\hat{f}+\hat{u}_{M}^{*}-\left(1+P_{M}^{\varepsilon}\right) \hat{y}_{d, 2}}{\left(1+P_{M}^{\varepsilon}\right)^{2}}-\frac{\hat{f}+\hat{u}_{\varepsilon, 0}^{*}-\left(1+\lambda_{0}^{\varepsilon}\right) \hat{y}_{d, 2}}{\left(1+\lambda_{0}^{\varepsilon}\right)^{2}}\right)\left(\overline{\hat{u}}_{\varepsilon, 0}^{*}-\hat{u}_{M}^{*}\right)\right](\eta) \mathrm{d} \eta \\
\leq & \mu_{2}\left(\sup _{\eta \in K}\left|\frac{1}{\left(1+P_{M}^{\varepsilon}(\eta)\right)^{2}}-\frac{1}{\left(1+\lambda_{0}^{\varepsilon}(\eta)\right)^{2}}\right|\right)\left\|\hat{u}_{\varepsilon, 0}^{*}-\hat{u}_{M}^{*}\right\|_{L^{2}(K)}\left\|\hat{f}+\hat{u}_{\varepsilon, 0}^{*}\right\|_{L^{2}(K ; \mathbb{C})} \\
& +\mu_{2}\left(\sup _{\eta \in K}\left|\frac{1}{1+\lambda_{0}^{\varepsilon}(\eta)}-\frac{1}{1+P_{M}^{\varepsilon}(\eta)}\right|\right)\left\|\hat{u}_{\varepsilon, 0}^{*}-\hat{u}_{M}^{*}\right\|_{L^{2}(K ; \mathbb{C})}\left\|\hat{y}_{d, 2}\right\|_{L^{2}(K ; \mathbb{C})} \\
\leq & C \varepsilon^{2 M}\left\|\hat{u}_{\varepsilon, 0}^{*}-\hat{u}_{M}^{*}\right\|_{L^{2}(K ; \mathbb{C})}\left(\left\|\hat{f}+\hat{u}_{\varepsilon, 0}^{*}\right\|_{L^{2}(K ; \mathbb{C})}+\left\|\hat{y}_{d, 2}\right\|_{L^{2}(K ; \mathbb{C})}\right) .
\end{aligned}
$$

Summing up,

$$
\kappa\left\|\hat{u}_{\varepsilon, 0}^{*}-\hat{u}_{M}^{*}\right\|_{L^{2}(K ; \mathbb{C})}^{2} \leq C \varepsilon^{2 M}\left(2\left\|\hat{f}+\hat{u}_{\varepsilon, 0}^{*}\right\|_{L^{2}(K ; \mathbb{C})}+\left\|\hat{y}_{d, 2}\right\|_{L^{2}(K ; \mathbb{C})}\right)\left\|\hat{u}_{\varepsilon, 0}^{*}-\hat{u}_{M}^{*}\right\|_{L^{2}(K ; \mathbb{C})} .
$$

This is the claimed result (3.3), since by Parseval's identity $\left\|\hat{u}_{\varepsilon, 0}^{*}\right\|_{L^{2}(K ; \mathbb{C})}=\left\|u_{\varepsilon}^{*}\right\|_{L^{2}\left(\mathbb{R}^{n}\right)}$, and $\left\|u_{\varepsilon}^{*}\right\|_{L^{2}\left(\mathbb{R}^{n}\right)}$ is uniformly bounded (Lem. 1.13).

Exploiting Parseval's identity along with (2.10) and (3.1), a straightforward consequence of Theorem 3.1 is the following approximation property for the optimal control of $\left(\mathrm{P}_{M}^{*}\right)$ :

Corollary 3.2. Let $\tilde{u}_{\varepsilon}^{*}$ be as in (2.10). Then the unique optimal control $u_{M}^{*}$ to $\left(\mathrm{P}_{M}^{*}\right)$ satisfies

$$
\left\|\tilde{u}_{\varepsilon}^{*}-u_{M}^{*}\right\|_{L^{2}\left(\mathbb{R}^{n}\right)} \leq C \varepsilon^{2 M}
$$

for some $\varepsilon$-independent constant $C>0$.

We next show that the solution $y_{M}^{*}$ of $(3.2)$ is the desired approximation of $\tilde{y}_{\varepsilon}^{*}$. 
Proposition 3.3 (Approximation of the solution to the PDE constraint). Let $y_{M}^{*}$ denote the solution to the effective equation (3.2) and let $\tilde{y}_{\varepsilon}^{*}$ be as in (2.11). Then

$$
\left\|\tilde{y}_{\varepsilon}^{*}-y_{M}^{*}\right\|_{L^{2}\left(\mathbb{R}^{n}\right)} \leq C \varepsilon^{2 M}
$$

for an $\varepsilon$-independent constant $C$.

Proof. In Proposition 2.4 we have already shown that the following function

$$
\tilde{y}_{M, a p p}^{*}(x)=\int_{K} \frac{\hat{f}(\eta)+\hat{u}_{\varepsilon, 0}^{*}(\eta)}{1+P_{M}^{\varepsilon}(\eta)} e^{2 \pi i \eta \cdot x} \mathrm{~d} \eta
$$

satisfies $\left\|\tilde{y}_{\varepsilon}^{*}-\tilde{y}_{M, a p p}^{*}\right\|_{L^{2}\left(\mathbb{R}^{n}\right)} \leq C \varepsilon^{2 M}$. By triangle inequality it is sufficient to prove

$$
\left\|\tilde{y}_{M, a p p}^{*}-y_{M}^{*}\right\|_{L^{2}\left(\mathbb{R}^{n}\right)} \leq C \varepsilon^{2 M} .
$$

Indeed, $\tilde{y}_{M, a p p}^{*}$ solves the effective equation with right hand side $f+\tilde{u}_{\varepsilon}^{*}$, while $y_{M}^{*}$ solves the same equation with right hand side $f+u_{M}^{*}$. By Corollary 3.2 one has $\left\|\left(f+\tilde{u}_{\varepsilon}^{*}\right)-\left(f+u_{M}^{*}\right)\right\|_{L^{2}\left(\mathbb{R}^{n}\right)}=\left\|\tilde{u}_{\varepsilon}^{*}-\tilde{u}_{M}^{*}\right\|_{L^{2}\left(\mathbb{R}^{n}\right)} \leq C \varepsilon^{2 M}$. Exploiting the linearity of the effective equation and the a priori estimate (2.21) from Proposition 2.6, one directly concludes

$$
\left\|\tilde{y}_{M, a p p}^{*}-\tilde{y}_{M}^{*}\right\|_{L^{2}\left(\mathbb{R}^{n}\right)} \leq\left\|\tilde{u}_{\varepsilon}^{*}-u_{M}^{*}\right\|_{L^{2}\left(\mathbb{R}^{n}\right)} \leq C \varepsilon^{2 M},
$$

which was the claim.

We conclude this section by discussing high-order approximations of the adjoint state corresponding to the optimal control problem $\left(\mathrm{P}_{\varepsilon}\right)$. By standard arguments $(c f .[33])$, the adjoint state $p_{\varepsilon}^{*}$ is characterized through the following adjoint equation:

$$
-\nabla \cdot\left(A^{\varepsilon} \nabla p_{\varepsilon}^{*}\right)+p_{\varepsilon}^{*}=\mu_{1}\left(-\nabla \cdot\left(A^{\varepsilon} \nabla\left(y_{\varepsilon}^{*}-y_{d, 1}^{\varepsilon}\right)+\left(y_{\varepsilon}^{*}-y_{d, 1}^{\varepsilon}\right)\right)+\mu_{2}\left(y_{\varepsilon}^{*}-y_{d, 2}^{\varepsilon}\right) .\right.
$$

It can therefore be written as a sum

$$
p_{\varepsilon}^{*}=\mu_{1}\left(y_{\varepsilon}^{*}-y_{d, 1}^{\varepsilon}\right)+\mu_{2} p_{\varepsilon, 1}^{*},
$$

where $p_{\varepsilon, 1}^{*}$ solves

$$
-\nabla \cdot\left(A^{\varepsilon} \nabla p_{\varepsilon, 1}^{*}\right)+p_{\varepsilon, 1}^{*}=y_{\varepsilon}^{*}-y_{d, 2}^{\varepsilon} .
$$

By Proposition 2.2 and (1.15), the right hand sides of (3.9) and (3.10) are nothing but $\mu_{1} \mathcal{A}^{\varepsilon}\left(\tilde{y}_{\varepsilon}^{*}-y_{d, 1}\right)+\mu_{2} p_{\varepsilon, 1}^{*}$ and $\mathcal{A}^{\varepsilon}\left(\tilde{y}_{\varepsilon}^{*}-y_{d, 2}\right)$. Therefore, analogously to the approximation results for $u_{\varepsilon}^{*}$ and $y_{\varepsilon}^{*}$, one can derive that

$$
\left\|p_{\varepsilon, 1}^{*}-\mathcal{A}^{\varepsilon}\left(p_{M}^{*}\right)\right\|_{L^{2}\left(\mathbb{R}^{n}\right)} \leq C \varepsilon^{2 M}
$$

for $p_{M}^{*}$ solving the $M$ th order approximate equation

$$
\sum_{k=1}^{M} \varepsilon^{2 k-2}(-1)^{k} \mathbb{A}_{2 k}^{*} D^{2 k} p_{M}^{*}+p_{M}^{*}=y_{M}^{*}-y_{d, 2} .
$$


Summing up, the following $M$ th order approximation result is obtained for the adjoint state:

$$
\left\|p_{\varepsilon}^{*}-\left(\mu_{1} \mathcal{A}^{\varepsilon}\left(y_{M}^{*}-y_{d, 1}\right)+\mu_{2} \mathcal{A}^{\varepsilon}\left(p_{M}^{*}\right)\right)\right\|_{L^{2}\left(\mathbb{R}^{n}\right)} \leq C \varepsilon^{2 M},
$$

whose proof is skipped for the sake of brevity.

\section{A WeLL-POSED EFFECTIVE PROBLEM}

The effective optimal control problem from Definition 1.15 is well-posed only for $\varepsilon$ lying below the threshold $\varepsilon_{M}$ introduced in Lemma 2.5. In this section we provide an alternative effective problem which does not require the smallness of $\varepsilon$. To keep the presentation simple and to demonstrate the main principle we restrict ourselves to second order approximations, i.e. $M=2$. However by an induction argument the analysis can be extended to higher-order approximations.

The central idea relies on the following formal calculation. For $M=2$ the effective PDE constraint (1.27) in one space dimension reads as

$$
-\mathbb{A}_{2}^{*} \partial_{x}^{2} y(x)+\varepsilon^{2} \mathbb{A}_{4}^{*} \partial_{x}^{4} y(x)+y(x)=f(x)+u(x),
$$

with $\mathbb{A}_{2}^{*}>0$ and $\mathbb{A}_{4}^{*} \leq 0$ as shown in [15]. We thus formally have $\mathbb{A}_{2}^{*} \partial_{x}^{2} y=y-f-u+O\left(\varepsilon^{2}\right)$. By rewriting $\varepsilon^{2} \mathbb{A}_{4}^{*} \partial_{x}^{4} y=\varepsilon^{2} \frac{\mathbb{A}_{4}^{*}}{\mathbb{A}_{2}^{*}} \partial_{x}^{2}\left(\mathbb{A}_{2}^{*} \partial_{x}^{2} y\right)$ and formally replacing $\mathbb{A}_{2}^{*} \partial_{x}^{2} y$ in (4.1) by $y-f-u$ we obtain the following effective equation

$$
-\left(\mathbb{A}_{2}^{*}-\varepsilon^{2} \frac{\mathbb{A}_{4}^{*}}{\mathbb{A}_{2}^{*}}\right) \partial_{x}^{2} y(x)+y(x)=\left(\operatorname{Id}+\varepsilon^{2} \frac{\mathbb{A}_{4}^{*}}{\mathbb{A}_{2}^{*}} \partial_{x}^{2}\right)(f(x)+u(x)),
$$

which is well-posed, since $\mathbb{A}_{2}^{*}-\varepsilon^{2} \frac{\mathbb{A}_{4}^{*}}{\mathbb{A}_{2}^{*}}>0$. In this simple form the above replacement procedure is known as the Boussinesq trick. In higher space dimension a similar, but more complicated calculation can be done. It has been shown in $[4,17]$ that every tensor $\mathbb{A}_{4}^{*} \in \mathbb{R}^{n \times n \times n \times n}$ allows for the following decomposition

$$
\mathbb{A}_{4}^{*} D^{4}=-\mathbb{B}_{2}^{*} D^{2}\left(\mathbb{A}_{2}^{*} D^{2}\right)+\mathbb{B}_{4}^{*} D^{4}
$$

with symmetric, positive semidefinite tensors $\mathbb{B}_{2}^{*} \in \mathbb{R}^{n \times n}$ and $\mathbb{B}_{4}^{*} \in \mathbb{R}^{n \times n \times n \times n}$. Proceeding exactly as in the one dimensional case we end up with the following effective PDE constraint

$$
-\left(\mathbb{A}_{2}^{*}+\varepsilon^{2} \mathbb{B}_{2}^{*}\right) D^{2} y(x)+\varepsilon^{2} \mathbb{B}_{4}^{*} D^{4} y(x)+y(x)=\left(\operatorname{Id}-\varepsilon^{2} \mathbb{B}_{2}^{*} D^{2}\right)(f(x)+u(x)),
$$

which is well-posed independently of the choice of $\varepsilon$. In the recent publication [3] the Boussinesq trick has been generalized to arbitrary order $M>2$ using an induction argument.

Proposition 4.1 (Well-posedness of the PDE constraint). Let $u \in L^{2}\left(\mathbb{R}^{n}\right)$ be a given control with Fourier transform $\hat{u}$ and $\operatorname{supp}(\hat{u}) \subset K$.

i) There exists a unique weak solution $y \in H^{1}\left(\mathbb{R}^{n}\right)$ to equation (4.3).

ii) The solution $y$ satisfies the a priori estimate

$$
\|y\|_{L^{2}\left(\mathbb{R}^{n}\right)}^{2}+2 \lambda\|\nabla y\|_{L^{2}\left(\mathbb{R}^{n}\right)}^{2} \leq C^{2}\|f+u\|_{L^{2}\left(\mathbb{R}^{n}\right)}^{2}
$$

with a constant $C$ depending only on $K$. Here $\lambda>0$ is the ellipticity constant of $\mathbb{A}_{2}^{*}$, i.e. such that $\mathbb{A}_{2}^{*} \cdot \eta^{\otimes 2} \geq \lambda|\eta|^{2}$ for every $\eta \in \mathbb{R}^{n}$. 
Proof. Consider

$$
\begin{aligned}
& Q_{2}^{\varepsilon}(\eta):=\varepsilon^{2}(2 \pi)^{2} \mathbb{B}_{2}^{*} \cdot \eta^{\otimes 2}, \\
& R_{2}^{\varepsilon}(\eta):=(2 \pi)^{2}\left(\mathbb{A}_{2}^{*}+\varepsilon^{2} \mathbb{B}_{2}^{*}\right) \cdot \eta^{\otimes 2}+\varepsilon^{2}(2 \pi)^{4} \mathbb{B}_{4}^{*} \cdot \eta^{\otimes 4} .
\end{aligned}
$$

By a direct calculation in Fourier space one finds that

$$
y(x):=\int_{K} \frac{1+Q_{2}^{\varepsilon}(\eta)}{1+R_{2}^{\varepsilon}(\eta)}(\hat{f}(\eta)+\hat{u}(\eta)) e^{2 \pi i \eta \cdot x} \mathrm{~d} \eta
$$

is a solution to (4.3). Moreover, every solution $\tilde{y}$ with Fourier transform $\hat{y}$ satisfies

$$
\left(1+R_{2}^{\varepsilon}(\eta)\right) \hat{y}(\eta)=\left(1+Q_{2}^{\varepsilon}(\eta)\right)(\hat{f}(\eta)+\hat{u}(\eta)) .
$$

Multiplying the above relation with $\hat{y}$, integrating over $\mathbb{R}^{n}$, taking into account $\hat{f}(\eta)=\hat{u}(\eta)=\hat{y}(\eta)=0$ for $\eta \notin K$, and exploiting that $\mathbb{B}_{2}^{*}$ and $\mathbb{B}^{*}{ }_{4}$ are positive semidefinite one finds

$$
\begin{aligned}
& \int_{\mathbb{R}^{n}}\left(1+\lambda|\eta|^{2}\right)|\hat{y}(\eta)|^{2} \mathrm{~d} \eta \leq \int_{K}\left(1+R_{2}^{\varepsilon}(\eta)\right)|\hat{y}(\eta)|^{2} \mathrm{~d} \eta \\
= & \int_{K}\left(1+Q_{2}^{\varepsilon}(\eta)\right)(\hat{f}(\eta)+\hat{u}(\eta)) \overline{\hat{y}}(\eta) \mathrm{d} \eta \leq C\|\hat{f}+\hat{u}\|_{L^{2}(K ; \mathbb{C})}\|\hat{y}\|_{L^{2}(K ; \mathbb{C})} \\
\leq & \frac{1}{2}\left(C^{2}\|\hat{f}+\hat{u}\|_{L^{2}(K ; \mathbb{C})}^{2}+\|\hat{y}\|_{L^{2}(K ; \mathbb{C})}^{2}\right) .
\end{aligned}
$$

The desired estimate (4.4) follows directly by Parseval's identity.

It is interesting to note how the prefactor $\frac{1+Q_{2}^{\varepsilon}}{1+R_{2}^{\varepsilon}}$ in $(4.5)$ is related to the former prefactors $\frac{1}{1+P_{2}^{\varepsilon}}$ and $\frac{1}{1+\lambda_{0}^{\varepsilon}}$, see (2.22) and (2.16). Actually

$$
\begin{aligned}
& \left(1+Q_{2}^{\varepsilon}(\eta)\right)\left(1+P_{2}^{\varepsilon}(\eta)\right)-\left(1+R_{2}^{\varepsilon}(\eta)\right) \\
= & \left(1+\varepsilon^{2}(2 \pi)^{2} \mathbb{B}_{2}^{*} \cdot \eta^{\otimes 2}\right)\left(1+(2 \pi)^{2} \mathbb{A}_{2}^{*} \cdot \eta^{\otimes 2}+\varepsilon^{2}(2 \pi)^{4} \mathbb{A}_{4}^{*} \cdot \eta^{\otimes 4}\right) \\
- & \left(1+(2 \pi)^{2}\left(\mathbb{A}_{2}^{*}+\varepsilon^{2} \mathbb{B}_{2}^{*}\right) \cdot \eta^{\otimes 2}+\varepsilon^{2}(2 \pi)^{4} \mathbb{B}_{4}^{*} \cdot \eta^{\otimes 4}\right)+O\left(\varepsilon^{4}\right) \\
= & \varepsilon^{2}(2 \pi)^{4}\left(\mathbb{B}_{2}^{*} \cdot \eta^{\otimes 2} \mathbb{A}_{2}^{*} \cdot \eta^{\otimes 2}+\mathbb{A}_{4}^{*} \cdot \eta^{\otimes 4}-\mathbb{B}_{4}^{*} \cdot \eta^{\otimes 4}\right)+O\left(\varepsilon^{4}\right) \stackrel{(4.2)}{=} O\left(\varepsilon^{4}\right),
\end{aligned}
$$

where the error is of order $\varepsilon^{4}$ uniformly in $\eta \in K$. In particular exploiting the fact that $R_{2}^{\varepsilon}$ and $\lambda_{0}^{\varepsilon}$ are nonnegative, we conclude that

$$
\begin{aligned}
\left|\frac{1+Q_{2}^{\varepsilon}(\eta)}{1+R_{2}^{\varepsilon}(\eta)}-\frac{1}{1+\lambda_{0}^{\varepsilon}(\eta)}\right| & =\left|\frac{\left(1+Q_{2}^{\varepsilon}(\eta)\right)\left(1+\lambda_{0}^{\varepsilon}(\eta)\right)-\left(1+R_{2}^{\varepsilon}(\eta)\right.}{\left(1+R_{2}^{\varepsilon}(\eta)\right)\left(1+\lambda_{0}^{\varepsilon}(\eta)\right)}\right| \\
& =\left|\frac{\left(1+Q_{2}^{\varepsilon}(\eta)\right)\left(1+P_{2}^{\varepsilon}(\eta)+O\left(\varepsilon^{4}\right)\right)-\left(1+R_{2}^{\varepsilon}(\eta)\right)}{\left(1+R_{2}^{\varepsilon}(\eta)\right)\left(1+\lambda_{0}^{\varepsilon}(\eta)\right)}\right| \\
& \leq\left|\left(1+Q_{2}^{\varepsilon}(\eta)\right)\left(1+P_{2}^{\varepsilon}(\eta)\right)-\left(1+R_{2}^{\varepsilon}(\eta)\right)\right|+O\left(\varepsilon^{4}\right) \\
& =O\left(\varepsilon^{4}\right)
\end{aligned}
$$

uniformly in $\eta \in K$. We emphasize once more that the advantage of working with $\frac{1+Q_{2}^{\varepsilon}}{1+R_{2}^{\varepsilon}}$ instead of $\frac{1}{1+P_{2}^{\varepsilon}}$ is that $1+R_{2}^{\varepsilon} \geq 1>0$ independently of $\varepsilon$, while this is not the case for $1+P_{2}^{\varepsilon}$. In view of (4.6), it is reasonable to 
introduce the following effective cost functional in Fourier form to obtain a second order approximation of the original cost functional $\hat{J}^{\varepsilon}$ from $(2.16)$.

Definition 4.2 (Well-posed effective optimization problem in Fourier form). Let $\hat{U}_{a d}$ be the effective admissible set from (2.23) and let $\mathbb{B}_{2}^{*}, \mathbb{B}_{4}^{*}$ be as in (4.2). Minimize the cost functional

$$
\begin{aligned}
\hat{I}_{2}^{*}(\hat{u})= & \frac{\mu_{1}}{2} \int_{K}\left|\frac{1+Q_{2}^{\varepsilon}(\eta)}{1+R_{2}^{\varepsilon}(\eta)}(\hat{f}(\eta)+\hat{u}(\eta))-\hat{y}_{d, 1}(\eta)\right|^{2} \frac{1+R_{2}^{\varepsilon}(\eta)}{1+Q_{2}^{\varepsilon}(\eta)} \mathrm{d} \eta \\
& +\frac{\mu_{2}}{2} \int_{K}\left|\frac{1+Q_{2}^{\varepsilon}(\eta)}{1+R_{2}^{\varepsilon}(\eta)}(\hat{f}(\eta)+\hat{u}(\eta))-\hat{y}_{d, 2}(\eta)\right|^{2} \mathrm{~d} \eta+\frac{\kappa}{2} \int_{K}|\hat{u}(\eta)|^{2} \mathrm{~d} \eta
\end{aligned}
$$

in the set $\hat{U}_{a d}$.

Let us comment on the above optimization problem. The problem is well-posed independently of $\varepsilon$ with a unique minimizer $\hat{u}_{2, \hat{I}}^{*} \in \hat{U}_{a d}$. Analogously to Theorem 3.1 we prove in Theorem 4.4 below that

$$
\left\|\hat{u}_{\varepsilon, 0}^{*}-\hat{u}_{2, \hat{I}}^{*}\right\|_{L^{2}(K ; \mathbb{C})} \leq C \varepsilon^{4} .
$$

Exactly as in Proposition 3.3 we then conclude, using the a priori estimate from Proposition 4.1, that

$$
y_{2, \hat{I}}^{*}(x):=\int_{K} \frac{1+Q_{2}^{\varepsilon}(\eta)}{1+R_{2}^{\varepsilon}(\eta)}\left(\hat{f}(\eta)+\hat{u}_{2, \hat{I}}^{*}(\eta)\right) e^{2 \pi i \eta \cdot x} \mathrm{~d} \eta
$$

satisfies the error estimate

$$
\left\|y_{\varepsilon}^{*}-y_{2, \hat{I}}^{*}\right\|_{L^{2}\left(\mathbb{R}^{n}\right)} \leq C \varepsilon^{4}
$$

for some $\varepsilon$-independent constant $C$. The optimization problem from Definition 4.2 is thus an alternative to approximate the original problem up to errors of order $\varepsilon^{4}$. Its central advantage lies in the fact that it is well-posed independently of $\varepsilon$.

Remark 4.3 (higher-order well-posed approximations).

i) As shown in [3] the Boussinesq trick can be generalized to arbitrary order $M \in \mathbb{N}$. This fact can be used to derive a higher-order effective optimal control problem that is well-posed independently of $\varepsilon$.

ii) In [7] a different regualarization approach of the effective equation (1.27) is proposed which relies on adding a high-order spatial derivative. [3] provides a brief comparision of both regularization methods.

We now prove the approximation property of the alternative effective optimization problem from Definition 4.2 .

Theorem 4.4 (Approximation of the optimal control). Let $\hat{u}_{2, \hat{I}}^{*} \in \hat{U}_{a d}$ be the unique minimizer of $\hat{I}_{2}^{*}$ and let $\hat{u}_{\varepsilon, 0}^{*} \in \hat{U}_{a d}$ be the unique minimizer of $\hat{J}^{\varepsilon}$. Then

$$
\left\|\hat{u}_{\varepsilon, 0}^{*}-\hat{u}_{2, \hat{I}}^{*}\right\|_{L^{2}(K ; \mathbb{C})} \leq C \varepsilon^{4} .
$$

for an $\varepsilon$-independent constant $C$.

Proof. The proof follows the lines of Theorem 3.1. With

$$
D \hat{I}_{2}^{*}(\hat{u}):=\mu_{1}\left(\frac{1+Q_{2}^{\varepsilon}}{1+R_{2}^{\varepsilon}}(\hat{f}+\hat{u})-\hat{y}_{d, 1}\right)+\mu_{2}\left(\frac{1+Q_{2}^{\varepsilon}}{1+R_{2}^{\varepsilon}}(\hat{f}+\hat{u})-\hat{y}_{d, 2}\right) \frac{1+Q_{2}^{\varepsilon}}{1+R_{2}^{\varepsilon}}+\kappa \hat{u},
$$


taking $\hat{u}_{\varepsilon, 0}^{*}$ as a test function in the variational inequality corresponding to $\hat{I}_{2}^{*}$ and $\hat{u}_{2, \hat{I}}^{*}$ as a test function in (3.4), one obtains

$$
\begin{aligned}
\int_{K} \operatorname{Re}[ & \left(\mu_{1}\left(\frac{1+Q_{2}^{\varepsilon}}{1+R_{2}^{\varepsilon}}\left(\hat{f}+\hat{u}_{2, \hat{I}}^{*}\right)-\hat{y}_{d, 1}\right)+\mu_{2}\left(\frac{1+Q_{2}^{\varepsilon}}{1+R_{2}^{\varepsilon}}\left(\hat{f}+\hat{u}_{2, \hat{I}}^{*}\right)-\hat{y}_{d, 2}\right) \frac{1+Q_{2}^{\varepsilon}}{1+R_{2}^{\varepsilon}}+\kappa \hat{u}_{2, \hat{I}}^{*}\right) \\
& \left.\times\left(\overline{\hat{u}_{\varepsilon, 0}^{*}}-\overline{\hat{u}_{2, \hat{I}}^{*}}\right)\right](\eta) \mathrm{d} \eta \geq 0
\end{aligned}
$$

and

$$
\int_{K} \operatorname{Re}\left[\left(\mu_{1}\left(\frac{\hat{f}+\hat{u}_{\varepsilon, 0}^{*}}{1+\lambda_{0}^{\varepsilon}}-\hat{y}_{d, 1}\right)+\mu_{2}\left(\frac{\hat{f}+\hat{u}_{\varepsilon, 0}^{*}}{1+\lambda_{0}^{\varepsilon}}-\hat{y}_{d, 2}\right)\left(1+\lambda_{0}^{\varepsilon}\right)^{-1}+\kappa \hat{u}_{\varepsilon, 0}^{*}\right)\left({\overline{\hat{u}_{2, \hat{I}}^{*}}}^{*}-\overline{\hat{u}}_{\varepsilon, 0}^{*}\right)\right](\eta) \mathrm{d} \eta \geq 0 .
$$

Summing up the two inequalities one concludes

$$
\begin{aligned}
& \kappa \int_{K}\left|\hat{u}_{\varepsilon, 0}^{*}(\eta)-\hat{u}_{2, \hat{I}}^{*}(\eta)\right|^{2}(\eta) \mathrm{d} \eta \\
\leq & \mu_{1} \int_{K} \operatorname{Re}\left[\left(\frac{1+Q_{2}^{\varepsilon}}{1+R_{2}^{\varepsilon}}\left(\hat{f}+\hat{u}_{2, \hat{I}}^{*}\right)-\frac{1}{1+\lambda_{0}^{\varepsilon}}\left(\hat{f}+\hat{u}_{\varepsilon, 0}^{*}\right)\right)\left(\overline{\hat{u}_{\varepsilon, 0}^{*}}-\overline{\hat{u}_{2, \hat{I}}^{*}}\right)\right](\eta) \mathrm{d} \eta \\
& +\mu_{2} \int_{K} \operatorname{Re}\left[\left(\left(\frac{1+Q_{2}^{\varepsilon}}{1+R_{2}^{\varepsilon}}\left(\hat{f}+\hat{u}_{2, \hat{I}}^{*}\right)-\hat{y}_{d, 2}\right) \frac{1+Q_{2}^{\varepsilon}}{1+R_{2}^{\varepsilon}}-\left(\frac{\hat{f}+\hat{u}_{\varepsilon, 0}^{*}}{1+\lambda_{0}^{\varepsilon}}-\hat{y}_{d, 2}\right)\left(1+\lambda_{0}^{\varepsilon}\right)^{-1}\right)\left(\hat{u}_{\varepsilon, 0}^{*}-\hat{u}_{2, \hat{I}}^{*}\right)\right](\eta) \mathrm{d} \eta .
\end{aligned}
$$

Since by (4.6) the difference between $\frac{1+Q_{2}^{\varepsilon}}{1+R_{2}^{\varepsilon}}$ and $\frac{1}{1+\lambda_{0}^{\varepsilon}}$ is of order $O\left(\varepsilon^{4}\right)$ uniformly in $\eta \in K$, we can argue exactly as in the proof of Theorem 3.1 to find

$$
\kappa\left\|\hat{u}_{\varepsilon, 0}^{*}(\eta)-\hat{u}_{2, \hat{I}}^{*}\right\|_{L^{2}(K ; \mathbb{C})}^{2} \leq C \varepsilon^{4}\left\|\hat{u}_{\varepsilon, 0}^{*}(\eta)-\hat{u}_{2, \hat{I}}^{*}\right\|_{L^{2}(K ; \mathbb{C})},
$$

which is the claimed result.

We conclude this section by rewriting the minimization problem from Definition 4.2 as an optimal control problem with PDE constraint (4.3). Unfortunately, the factor $\frac{1+R_{2}^{\varepsilon}(\eta)}{1+Q_{2}^{\varepsilon}(\eta)}$ in the first part of the cost functional $\hat{I}_{2}^{*}$ does not allow for a simple representation of the cost functional in the corresponding optimal control problem.

Proposition 4.5 (Reformulation as an optimal control problem). The minimization problem from Definition 4.2 is equivalent to minimizing the cost functional

$$
\begin{aligned}
I_{2}^{*}(u, y, w):=\frac{\mu_{1}}{2} & \int_{\mathbb{R}^{n}}\left(\left\langle D\left(w-w_{d, 1}\right)(x), D\left(w-w_{d, 1}\right)(x)\right\rangle_{\mathbb{A}_{2}^{*}+2 \varepsilon^{2} \mathbb{B}_{2}^{*}}\right. \\
& +\varepsilon^{2}\left\langle D^{2}\left(w-w_{d, 1}\right)(x), D^{2}\left(w-w_{d, 1}\right)(x)\right\rangle_{\mathbb{B}_{4}^{*}+\mathbb{A}_{2}^{*} \mathbb{B}_{2}^{*}+\varepsilon^{2} \mathbb{B}_{2}^{*} \mathbb{B}_{2}^{*}} \\
& \left.+\varepsilon^{4}\left\langle D^{3}\left(w-w_{d, 1}\right)(x), D^{3}\left(w-w_{d, 1}\right)(x)\right\rangle_{\mathbb{B}_{4}^{*} \mathbb{B}_{2}^{*}}+\left|w(x)-w_{d, 1}(x)\right|^{2}\right) \mathrm{d} x \\
& +\frac{\mu_{2}}{2} \int_{\mathbb{R}^{n}}\left|y(x)-y_{d, 2}(x)\right|^{2} \mathrm{~d} x+\frac{\kappa}{2} \int_{\mathbb{R}^{n}}|u(x)|^{2} \mathrm{~d} x
\end{aligned}
$$

in the set $U_{a d}^{*}$ under the PDE constraint (4.3). The functions $w, w_{d, 2}$ are determined through

$$
-\varepsilon^{2} \mathbb{B}_{2}^{*} D^{2} w(x)+w(x)=y(x) \quad \text { and } \quad-\varepsilon^{2} \mathbb{B}_{2}^{*} D^{2} w_{d, 2}(x)+w_{d, 2}(x)=y_{d, 2}(x),
$$

where the composition of tensors is defined as 


$$
\begin{aligned}
\left(\mathbb{A}_{2}^{*} \mathbb{B}_{2}^{*}\right)_{i_{1}, i_{2}, i_{3}, i_{4}} & :=\left(\mathbb{A}_{2}^{*}\right)_{i_{1}, i_{3}}\left(\mathbb{B}_{2}^{*}\right)_{i_{2}, i_{4}} \quad \text { and } \quad\left(\mathbb{B}_{2}^{*} \mathbb{B}_{2}^{*}\right)_{i_{1}, i_{2}, i_{3}, i_{4}}:=\left(\mathbb{B}_{2}^{*}\right)_{i_{1}, i_{3}}\left(\mathbb{B}_{2}^{*}\right)_{i_{2}, i_{4}} \\
\left(\mathbb{B}_{4}^{*} \mathbb{B}_{2}^{*}\right)_{i_{1}, \ldots, i_{6}} & :=\left(\mathbb{B}_{4}^{*}\right)_{i_{1}, i_{2}, i_{4}, i_{5}}\left(\mathbb{B}_{2}^{*}\right)_{i_{3}, i_{6}} .
\end{aligned}
$$

In particular $\hat{u}_{2, \hat{I}}^{*}$ is the Fourier transform of the unique optimal control $u_{2, I}^{*}$.

Note that the above definition is designed in such way that the composition of two symmetric tensors is again symmetric. Hereby symmetry is understood in weak sense. We say that a 4 -tensor $\mathbb{A} \in \mathbb{R}^{n^{4}}$ and a 6 -tensor $\mathbb{B} \in \mathbb{R}^{n^{6}}$ are symmetric, if $(\mathbb{A})_{i_{1}, i_{2}, i_{3}, i_{4}}=(\mathbb{A})_{i_{3}, i_{4}, i_{1}, i_{2}}$ and $(\mathbb{B})_{i_{1}, i_{2}, i_{3}, i_{4}, i_{5}, i_{6}}=(\mathbb{B})_{i_{4}, i_{5}, i_{6}, i_{1}, i_{2}, i_{3}}$ for all indeces $i_{1}, \ldots, i_{6} \in\{1, \ldots, n\}$.

Proof of Proposition 4.5. The second and third part of the energy are clear due to Parseval's identity. It remains to justify the first part of $I_{2}^{*}$. Indeed, denoting the Fourier transform of $\tilde{y}$ by $\hat{y}$, the first part on the cost functional $\hat{I}_{2}^{*}$ from Definition 4.2 reads as

$$
\begin{aligned}
& \frac{\mu_{1}}{2} \int_{K}\left|\frac{1+Q_{2}^{\varepsilon}(\eta)}{1+R_{2}^{\varepsilon}(\eta)}(\hat{f}(\eta)+\hat{u}(\eta))-\hat{y}_{d, 1}(\eta)\right|^{2} \frac{1+R_{2}^{\varepsilon}(\eta)}{1+Q_{2}^{\varepsilon}(\eta)} \mathrm{d} \eta \\
= & \frac{\mu_{1}}{2} \int_{K}\left|\hat{y}(\eta)-\hat{y}_{d, 1}(\eta)\right|^{2} \frac{1+R_{2}^{\varepsilon}(\eta)}{1+Q_{2}^{\varepsilon}(\eta)} \mathrm{d} \eta \\
= & \frac{\mu_{1}}{2} \int_{K}\left|\frac{\hat{y}(\eta)-\hat{y}_{d, 1}(\eta)}{1+Q_{2}^{\varepsilon}(\eta)}\right|^{2}\left(1+R_{2}^{\varepsilon}(\eta)\right)\left(1+Q_{2}^{\varepsilon}(\eta)\right) \mathrm{d} \eta .
\end{aligned}
$$

By a direct calculation in Fourier space one finds that $\frac{\hat{y}(\eta)}{1+Q_{2}^{\varepsilon}(\eta)}$ is the Fourier transform of $w$ and $\frac{\hat{y}_{d, 1}(\eta)}{1+Q_{2}^{\varepsilon}(\eta)}$ the Fourier transform of $w_{d, 2}$. Moreover,

$$
\begin{aligned}
& \left(1+R_{2}^{\varepsilon}(\eta)\right)\left(1+Q_{2}^{\varepsilon}(\eta)\right) \\
= & 1+(2 \pi)^{2}\left[\mathbb{A}_{2}^{*}+2 \varepsilon^{2} \mathbb{B}_{2}^{*}\right] \cdot \eta^{\otimes 2}+\varepsilon^{2}(2 \pi)^{4}\left[\mathbb{B}_{4}^{*}+\mathbb{A}_{2}^{*} \mathbb{B}_{2}^{*}+\varepsilon^{2} \mathbb{B}_{2}^{*} \mathbb{B}_{2}^{*}\right] \cdot \eta^{\otimes 4}+\varepsilon^{4}(2 \pi)^{6} \mathbb{B}_{4}^{*} \mathbb{B}_{2}^{*} \cdot \eta^{\otimes 6},
\end{aligned}
$$

which, together with Parseval's identity, provides the claimed result.

\section{REFERENCES}

[1] A. Abdulle and T.N. Pouchon, A priori error analysis of the finite element heterogeneous multiscale method for the wave equation in heterogeneous media over long time. SIAM J. Numer. Anal. 54 (2016) 1507-1534.

[2] A. Abdulle and T.N. Pouchon, Effective models for the multidimensional wave equation in heterogeneous media over long time and numerical homogenization. Math. Models Methods Appl. Sci. 26 (2016).

[3] A. Abdulle and T.N. Pouchon, Effective models and numerical homogenization for wave propagation in heterogeneous media on arbitrary timescales. Found. Comput. Math. 20 (2020) 1505-1547.

[4] G. Allaire, M. Briane and M. Vanninathan, A comparison between two scale asymptotic expansions and Bloch wave expansions for the homogenization of periodic structures. SEMA J. 73 (2016) 237-259.

[5] G. Allaire, A. Lamacz-Keymling and J. Rauch, Crime pays; homogenized wave equations for long times. To appear Asymptotic Analysis.

[6] N. Bakhvalov and G. Panasenko, Homogenization: Averaging Processes in Periodic Media. Kluwer, Dordrecht (1989).

[7] A. Benoit and A. Gloria, Long-time homogenization and asymptotic ballistic transport of classical waves. Annales Scientifiques de l'École Normale Supérieure 52 (2019) 703-759.

[8] A. Bensoussan, J.-L. Lions and G. Papanicolaou, Asymptotic analysis for periodic structures. Corrected reprint of the 1978 original, AMS Chelsea Publishing, Providence, RI (2011).

[9] G. Bouchitté and B. Schweizer, Homogenization of Maxwell's equations in a split ring geometry. Multiscale Model. Simul. 8 (2010) 717-750.

[10] S. Brahim-Otsmane, G.A. Francfort and F. Murat, Correctors for the homogenization of the wave and heat equations. J. Math. Pures Appl. 71 (1992) 197-231.

[11] G. Buttazzo, M.E. Drakhlin, L. Freddi and E. Stepanov, Homogenization of optimal control problems for functional-differential equations. J. Optim. Theory Appl. 93 (1997) 103-119.

[12] D. Cioranescu, A. Damlamian, P. Donato, G. Griso and R. Zaki, The periodic unfolding method in domains with holes. SIAM J. Math. Anal. 44 (2012) 718-760. 
[13] C. Conca and M. Vanninathan, Homogenization of periodic structures via Bloch decomposition. SIAM J. Appl. Math. 57 (1997) 1639-1659.

[14] C. Conca, R. Orive and M. Vanninathan, Bloch approximation in homogenization and applications. SIAM J. Math. Anal. 33 (2002) 1166-1198.

[15] C. Conca, R. Orive and M. Vanninathan, On Burnett coefficients in periodic media. J. Math. Phys. 47 (2006) 032902.

[16] U. De Maio, P. Kogut and R. Manzo, Asymptotic analysis of an optimal boundary control problem for ill-posed elliptic equation in domains with rugous boundary. Asymptot. Anal. 118 (2020) 209-234.

[17] T. Dohnal, A. Lamacz and B. Schweizer, Bloch-wave homogenization on large time scales and dispersive effective wave equations. Multiscale Model. Simul. 12 (2014) 488-513.

[18] T. Dohnal, A. Lamacz and B. Schweizer, Dispersive homogenized models and coefficient formulas for waves in general periodic media. Asymptot. Anal. 93 (2015) 21-49.

[19] S. Kesavan and T. Muthukumar, Homogenization of an optimal control problem with state-constraints. Differ. Equ. Dyn. Syst. 19 (2011) 361-374.

[20] S. Kesavan and M. Rajesh, Homogenization of periodic optimal control problems via multi-scale convergence. Proc. Indian Acad. Sci. Math. Sci. 108 (1998) 189-207.

[21] S. Kesavan and J. Saint Jean Paulin, Homogenization of an optimal control problem. SIAM J. Control Optim. 35 (1997) $1557-1573$.

[22] P. Kogut, Higher-order asymptotics of the solutions of the problem of the optimal control of a distributed system with rapidly oscillating coefficients. Ukrainian Math. J. 48 (1996) 1063-1073.

[23] P. Kogut and G. Leugering, Homogenization of optimal control problems in variable domains. Principle of the fictitious homogenization. Asymptot. Anal. 26 (2001) 37-72.

[24] P. Kogut and G. Leugering, On S-homogenization of an optimal control problem with control and state constraints. Z. Anal. Anwen. 20 (2001) 395-429.

[25] P. Kogut and G. Leugering, Asymptotic analysis of state constrained semilinear optimal control problems. J. Optim. Theory Appl. 135 (2007) 301-321.

[26] P. Kogut and G. Leugering, Homogenization of Dirichlet optimal control problems with exact partial controllability constraints. Asymptot. Anal. 57 (2008) 229-249.

[27] P. Kogut and G. Leugering, Optimal control problems for partial differential equations on reticulated domains. Approximation and asymptotic analysis. Systems 83 Control: Foundations 85 Applications. Birkhäuser/Springer, New York (2011).

[28] A. Lamacz, Dispersive effective models for waves in heterogeneous media. Math. Models Methods Appl. Sci. 21 (2011) 18711899.

[29] A. Lamacz and B. Schweizer, Effective acoustic properties of a meta-material consisting of small Helmholtz resonators. Discrete Contin. Dyn. Syst. Ser. S 10 (2017) 815-835.

[30] J.-L. Lions, Contrôle optimal de systèmes gouvernés par des équations auxdérivées partielles. Dunod, Paris; Gauthier-Villars, Paris (1968).

[31] E. Sanchez-Palencia, Non-Homogeneous Media and Vibration Theory. Vol. 120 of Springer Lecture Notes in Physics (1980).

[32] F. Santosa and W. Symes, A dispersive effective medium for wave propagation in periodic composites. SIAM J. Appl. Math. 51 (1991) 984-1005.

[33] F. Tröltzsch, Optimal Control of Partial Differential Equations. Grad. Stud. Math. 112. AMS, Providence, RI (2010).

[34] I. Yousept, Optimal control of quasilinear $\boldsymbol{H}$ (curl)-elliptic partial differential equations in magnetostatic field problems. SIAM J. Control and Optim. 51 (2013) 3624-3651.

[35] I. Yousept, Optimal control of non-smooth hyperbolic evolution Maxwell equations in type-II superconductivity. SIAM J. Control and Optim. 55 (2017) 2305-2332.

\section{Subscribe to Open (S20) A fair and sustainable open access model}

This journal is currently published in open access under a Subscribe-to-Open model (S2O). S2O is a transformative model that aims to move subscription journals to open access. Open access is the free, immediate, online availability of research articles combined with the rights to use these articles fully in the digital environment. We are thankful to our subscribers and sponsors for making it possible to publish this journal in open access, free of charge for authors.

\section{Please help to maintain this journal in open access!}

Check that your library subscribes to the journal, or make a personal donation to the $\mathrm{S} 2 \mathrm{O}$ programme, by contacting subscribers@edpsciences.org

More information, including a list of sponsors and a financial transparency report, available at: https://www.edpsciences.org/en/maths-s2o-programme 